\title{
Optimizing temporary work and overtime in the Time Cost Quality Trade-off Problem
}

\author{
Jully Jeunet $^{\mathrm{a}, *}$, Mayassa Bou Orm ${ }^{\mathrm{b}}$ \\ ${ }^{a}$ CNRS, Université Paris Dauphine, PSL Research University, CNRS UMR[7243], Lamsade, place du Maréchal de Lattre de Tassigny, 75775 Paris, France \\ ${ }^{\mathrm{b}}$ Université Paris Dauphine, PSL Research University, CNRS UMR[7243], Lamsade, place du Maréchal de Lattre de Tassigny, 75775 Paris, France
}

\section{A R T I C L E I N F O}

\section{Article history:}

Received 24 May 2018

Accepted 9 January 2020

Available online 17 January 2020

\section{Keywords:}

Project scheduling

Time Cost Quality Trade-off Problem

Activity quality

Temporary work

Overtime

\begin{abstract}
A B S T R A C T
In spite of its significant contribution to project success, quality has been scarcely addressed in the literature on deterministic project scheduling problems. Although it is recognized that higher qualities are associated with longer processing times, no relationship between quality and resource consumption has been analytically derived to support this statement. As manufacturing projects can be accelerated using additional manpower such as overtime and temporary workers, we derive an analytical relationship between quality and manpower since overtime and overmanning negate quality. We also take into account productivity losses due to overmanning. Contrary to most previous contributions that focus on the project overall quality as an aggregation of quality levels attained at the individual activities, we impose each activity to reach a minimum quality threshold, which is consistent with project management practices. Consequently, we develop a mixed integer linear programming (MILP) to optimize temporary work and overtime so as to accelerate a project with quality and productivity considerations. The objective is to simultaneously determine for each activity the number of permanent, temporary and overtime workers over the processing periods in order to minimize the makespan, the total cost and the overall quality losses subject to individual quality constraints, precedence relationships, nonpreemption and availability of resources. Our approach is successfully applied on numerous instances based on a real project of a high speed locomotive as well as on other projects taken from the literature.
\end{abstract}

(c) 2020 Elsevier B.V. All rights reserved.

\section{Introduction}

The Time Cost Trade-off Problem (TCTP) has been widely studied in the literature on deterministic project scheduling problems (for a survey, see Weglarz, Jozefowska, Mika, \& Waligora, 2011 or Hartmann \& Briskorn, 2010). However, projects that are delivered on time within budget can hardly be considered as successful if the quality of the deliverable is poor. The reason is that quality products make an important contribution to long-term revenue and profitability and enable companies to maintain their reputation and customer loyalty. It is thus surprising that only a few references address the quality issue in the TCTP in spite of its significance (for a survey, see Orm \& Jeunet, 2018).

A project schedule often needs to be shortened to fit within contractually time. This almost always requires additional manpower, namely overtime and temporary work, since the majority of manufacturing systems are not fully automatized. As a result,

\footnotetext{
* Corresponding author.

E-mail addresses: jully.jeunet@dauphine.fr (J. Jeunet) mayassa.bouorm@lamsade.dauphine.fr (M. Bou Orm).
}

cost increases and quality declines. Overtime implies fatigue that affects product quality (Babu \& Suresh, 1996; Beckers et al., 2004; Hanna \& Sullivan, 2004; Khang \& Myint, 1999; Li, Love, \& Drew, 2000; Mahmud, 2015). Temporary workers favor overmanning which also negates quality (Hanna, Chang, Lackney, \& Sullivan, 2007; Li et al., 2000). Besides, it is notorious that overtime leads to productivity losses (Broschak \& Davis-Blake, 2006; Gunduz, 2004; Hanna \& Sullivan, 2004; Kaming, Holt, Kometa, \& Olomolaiye, 1998). Furthermore, overmanning also has a negative impact on productivity (Gibson, 2015; Gunduz, 2004; Gunduz \& Hanna, 2005; Hitchens, Wagner, \& Birnie, 1992; Kaming et al., 1998). Considering the adverse effects of additional manpower on quality and on productivity, the question arises how to plan manpower requirements so as to accelerate a manufacturing project at the lowest possible cost while meeting quality constraints on activities?

To address this research question, we develop a tactical manpower planning model with the objectives of minimizing the makespan, the total cost of manpower and overall quality losses of a manufacturing project, subject to manpower requirements, precedence and nonpreemption constraints, human resource availability and minimum quality levels for activities. We therefore 
deal with the Time Cost Quality Trade-off problem where the resources are limited to three types of manpower: permanent workers, overtime, and temporary workers since in the mid-run, tools and equipment remain unchanged. We assume that each activity requires a single specific skill, like welding or cabling and each worker is endowed with a single skill. This is consistent for most manufacturing projects contrary to services projects. A worker can process only one activity at a time but several workers with the same skill can simultaneously process one activity. The completion of any activity requires a work content expressed in man-days that must be met by a proper allocation of workers over the processing periods, namely the work profile (Fundeling \& Trautmann, 2010). Since the additional manpower is inherently flexible, the work profile is assumed to be flexible, meaning that the number of workers allocated to an activity can vary from one processing period to the next. For instance, a work content of 12 man-days for a given activity with a constant profile of 2 men for 6 days (normal duration) can be completed in 4 days with a flexible profile of 4 men for 2 days and 2 men for 2 days. In the latter case, there is overmanning for 2 days which may result in productivity losses. We therefore provide a new formulation of the work content constraint to account for productivity losses. When accelerated, an activity experiences a quality decline due to the additional manpower. We analytically derive the impact of overtime and temporary work on quality that we incorporate into the constraints of minimum quality levels. The planning problem is formulated as a Mixed Integer Linear Program for optimizing the allocation of permanent and temporary workers as well as overtime hours to each processing period of every activities.

Examining the Time Cost Quality trade-off in a project management setting appears to be an understudied area in the Operations Management literature relative to its importance in practice. Quality refers to the degree to which products fulfill a number of requirements that have been deemed necessary. In most contributions, quality is measured by an indicator between zero and one reflecting the percentage of validated items in quality control inspection lists to verify compliance with the applicable contract requirements (Orm \& Jeunet, 2018). Tareghian and Taheri (2006) incorporate such a quality indicator into a Discrete Time Cost Trade-off Problem (DTCTP) with a single non-renewable resource where each activity can be completed in several execution modes. Each mode is assigned with an estimated cost, duration and quality. The objective is to find modes for activities so as to minimize the makespan or to minimize the cost or to maximize the overall quality expressed as the mean of individual qualities. Thus, they develop three integer programming models where each model optimizes one of the given objectives under desired bounds constraints on the other two (epsilon constraint method). Afruzi, Najafi, Roghanian, and Mazinani (2014) extend this approach to a multiple resources setting where each activity can be executed either in a normal mode (minimum allocation of resources) or in a crashed mode (maximum allocation of resources) with dependent mode-activity assignments: the choice of a mode for a given activity implies that successor activities are completed in the same mode. The authors develop a Multi-Objective Imperialist Competitive Algorithm to find the best compromises between time, cost and quality of the project under resources and mode identity constraints. The project overall quality is expressed as a weighted sum of individual qualities. In order to be applicable to real case problems, both previous approaches thus imply an estimation of duration, cost and quality for each activity in their numerous execution modes. Only Babu and Suresh (1996) formulate the quality of an activity as a linear function of duration and since duration is also assumed to decrease linearly with a single non-renewable resource, thus quality is a linear increasing function of this single resource (cost). Consequently the authors implicitly assumed that increasing the resources has no impact on productivity. Like in Tareghian and Taheri (2006), three linear programming models are developed to optimize one of these entities by assigning desired bounds on the other two, with decision variables consisting of activity completion times. Again, the overall project quality results from an aggregation of quality levels attained at the individual activities.

The quality criterion has never been integrated into the Multiple Discrete Time Resources Trade-off Problem (MDTRTP) which itself remains understudied in the literature. Naber and Kolisch (2014) address the Resource-Constrained Project Scheduling Problem with flexible resource profiles (FRCPSP), where the objective is to determine start times and resource profiles for all activities so as to minimize the makespan, subject to precedence relationships and resource constraints.

Besides, optimization of temporary work and overtime has received little attention in the literature on project scheduling. Yang, Guenes, and Brien (2004) tackle the single resource TCTP where jobs can be processed in regular and overtime modes. The objective is to minimize the total cost of overtime and tardiness under precedence and nonpreemption constraints. Heimerl and Kolisch (2010) consider not only overtime but also temporary work for simultaneously scheduling independent projects requiring multiskilled human resources with the objective of minimizing labor costs. Thus, each worker masters several skills, and workers may perform differently with respect to a same skill (heterogeneous efficiencies) with no learning or forgetting effect (static efficiencies).

To the best of our knowledge, our paper is the first to integrate quality into an optimization model for additional manpower allocation to accelerate a manufacturing project. We consider not only overtime but also temporary work and their impact on quality and on productivity. In this regard, we provide a thorough analysis of these impacts as well as their mathematical formulation. None of the existing contributions formulates the quality of an activity as a function of several renewable resources whereas we provide a mechanism for modeling this relationship. Thus, with very few parameters to estimate for each activity, we can obtain the cost, the quality and the duration associated with an almost infinite number of execution modes. This makes the implementation of our approach in practice much easier than multi-mode modeling approaches that require an estimation of parameters in each activity execution mode. Besides, existing contributions focus on the maximization of the overall quality of a project defined as an aggregation of individual qualities. But such an aggregation has an inherent flaw: the project quality can be acceptable with some unacceptable individual qualities as long as they are offset by other high individual qualities (see Babu \& Suresh, 1996 or Khang \& Myint, 1999). In practice however, the quality of each activity must reach a minimum level which may be estimated by the rate of unacceptable flaws in the checklist. We thus include in our model minimum quality constraints on each activity.

Contrary to existing papers that adopt either an epsilon constraint method or a multi-objective approach, we use a lexicographic optimization method where the makespan is to be minimized first, then the total cost and ultimately the overall quality losses while meeting individual quality constraints. This preference order of the objectives is consistent with managers goals (Mungle, Benyoucef, Son, \& Tiwari, 2013). The model is successfully applied to a case study based on a real manufacturing project of a very high speed train motor coach at Alstom, the French company operating worldwide in rail transport markets. In addition, several realistic instances from the extant literature were also used to validate the performance of our model.

The paper is organized as follows. Section 2 describes our planning problem and gives insights about the effects of additional manpower on processing times and on quality. In Section 3, 
these effects are discussed in detail and analytically derived to achieve the formulation of our planning problem. Section 4 is dedicated to the numerical results. Finally, Section 5 summarizes our contribution and outlines directions for future work.

\section{Problem description}

In this Section, we state the planning problem and we briefly depict the impacts of additional manpower.

We consider a project represented as an Activity-on-Node graph with a set of activities $V=\{0,1, \ldots, n, n+1\}$ where 0 and $n+1$ are dummy activities denoting respectively the start and finish of the project. Arcs represent precedence relationships between activities; each activity $j$ having a set $\Gamma^{-1}(j)$ of predecessor activities and a set $\Gamma(j)$ of successors. Each activity requires a single skill $k$, with $k=1, \ldots, K$ where $K$ is the total number of different skills needed to complete the project and $K \leq n$. Workers are each endowed with a single skill and $\mathcal{P}_{k}$ denotes the number of workers with skill $k$ allocated to the project. We let $V_{k}$ be the subset of activities requiring skill $k$. When the project is not accelerated or said to be executed in a normal mode, its makespan $C_{\max }^{\text {nor }}$ is obtained by allocating to each activity $j$ a number $P_{j}^{\text {nor }}$ of workers with skill $k$ in each period of its normal processing time $d_{j}^{\text {nor }}$. The work content in man-days of activity $j$ is thus equal to $P_{j}^{\text {nor }} \cdot d_{j}^{\text {nor }}$. For dummy activities, we have $d_{0}^{\text {nor }}=d_{n+1}^{\text {nor }}=0$.

A lower bound $C_{\max }$ on the makespan can be derived from lower bounds $\left\{\underline{d}_{j}\right\}_{j=1, \ldots, n}$ on activities durations obtained by allocating to each activity the maximum number of workers and overtime hours in each processing period. We let $E S_{j}$ be the earliest start time of activity $j$ based on lower bounds $\left\{\underline{d}_{j}\right\}_{j=1, \ldots, n}$ and $L F_{j}$, its latest finish time derived from upper bounds $\left\{d_{j}^{\text {nor }}\right\}_{j=1, \ldots, n}$. We define $\mathcal{T}_{j}=\left\{E S_{j}, \ldots, L F_{j}\right\}$ as the set of feasible processing periods of activity $j$.

The objective is to sequentially minimize the project makespan, the total cost and the overall quality losses by optimizing the permanent and temporary workforce and overtime subject to work content satisfaction for each activity, precedence relationships, nonpreemption, resource availability and individual qualities above acceptable thresholds. Decision variables $p_{j t}, u_{j t}$ and $h_{j t}$ denote respectively the number of permanent workers, temporary workers and overtime hours assigned to the execution of activity $j$ in period $t$. Additionally, $z_{j t}$ is a binary variable that takes a value of 1 if activity $j$ is being processed in period $t$ and 0 otherwise. Thus, after optimization, the duration $d_{j}$ of an activity $j$ is $d_{j}=\sum_{t \in \mathcal{T}_{j}} z_{j t}$. The notations and definitions are summarized in Table 5 in Appendix A.

Fig. 1 provides a brief description of the planning problem on the left hand side, and gives an overview of the impacts of additional manpower on the duration and on the quality on the right hand side. Each of these impacts is studied in detail in Section 3 as indicated in Fig. 1. The influence of additional manpower on the duration is examined in Section 3.1, which corresponds to the blue arrows (solid line) in the figure. Temporary work favors overmanning and hence productivity losses that modify the formulation of the work profile, as detailed in Section 3.1.1. Overtime also leads to productivity losses beyond a threshold of hours as discussed in Section 3.1.2. We finally obtain a formulation of the work profile that, together with the work content, allow for deriving the relationship between manpower usage and duration (Section 3.1.3).

Next, the effects of additional manpower on quality are analyzed in Section 3.2 with individual impacts represented by the red arrows (dotted line) in Fig. 1. Temporary work negates quality through overmanning (Section 3.2.1). The negative impact of overtime on quality is mainly due to a decreased attention to quality standards and expectations at the end of the working day (Section 3.2.2). Since quality is formulated as a function of manpower and as manpower usage defines the processing time, we derive the relationship between quality and duration in Section 3.2.3.

\section{Analysis of the impacts of additional manpower and formulation}

In this Section, we first provide a formulation of the relationship between additional manpower and processing time (Section 3.1), by analyzing the impact of overmanning and overtime on productivity. Second, we derive the relationship between quality and additional manpower (Section 3.2.3), distinguishing again the effect of overmanning and overtime on quality. These relationships are finally incorporated into the formulation of the optimization program that we give in Section (3.3).

\subsection{Relationship between additional manpower and processing time}

Previous contributions assume a linear relationship between the processing time and the cost. Since the cost is proportional to the hours worked, this amounts to assume constant returns to scale (Babu \& Suresh, 1996 and Khang \& Myint, 1999). However, as documented in our introduction, several studies advocate that additional manpower causes productivity losses which result in decreasing returns to scale. The relationship between the processing time and the additional manpower is therefore convex as will be illustrated at the end of this Section. In order to analytically incorporate potential productivity losses in our model, the work content constraints need to be reformulated. These constraints state that the number of workers allocated to an activity over its processing periods must satisfy its work content, which is written as

$\sum_{t \in \mathcal{T}_{j}}\left(W_{j t}^{R}+W_{j t}^{O}\right)=P_{j}^{\text {nor }} \cdot d_{j}^{\text {nor }} \quad \forall j=1, \ldots, n$,

where $W_{j t}^{R}$ and $W_{j t}^{O}$ designate the number of productive workers during regular and overtime hours in period $t$ respectively. We have $W_{j t}^{R} \equiv W_{j}^{R}\left(p_{j t}+u_{j t}\right)$ and $W_{j t}^{O} \equiv W_{j}^{O}\left(h_{j t}\right)$ and the shapes of these functions are discussed in the next Subsections.

\subsubsection{Impact of overmanning on productivity}

Overmanning for activity $j$ in period $t$ is defined as the situation where the number of workers on this activity in regular working time exceeds the normal number $P_{j}^{\text {nor }}$ in that period, that is $p_{j t}+u_{j t}>P_{j}^{\text {nor }}$. Besides, capacity constraints on workstations limit overmanning to a maximum number of $P_{j}^{\max }$ workers (with skill $k$ ) in each period. Beyond $P_{j}^{\text {nor }}$, the workforce can include not only temporary workers but also permanent workers since some of them become available in some periods, as a result of acceleration. Thus we can have $p_{j t}+u_{j t}>P_{j}^{\text {nor }}$ with $p_{j t}>P_{j}^{\text {nor }}$.

We assume that workers with a same skill have a same productivity, be these workers permanent or temporary. First, most of the empirical literature finds that temporary workers are not less committed than permanent workers (for a survey, see Cuyper et al., 2008). Second, for the skilled work we consider here, a quite short training period is sufficient to guarantee a same productivity. Third, companies hire the same temporary workers as often as possible. Consequently, productivity is only affected by overmanning and not by heterogeneous skill levels among workers (permanent or temporary). Overmanning implies productivity losses due to limited workspace (site congestion), crew interference and dilution of supervision (Gunduz, 2004; Gunduz, 2004; Kaming et al., 1998 ) or because the presence of temporary workers may create conflict and tension in work groups (Broschak \& Davis-Blake, 2006). In their literature review, Hanna et al. (2007) concluded that all prior studies demonstrate a negative effect of overmanning on 


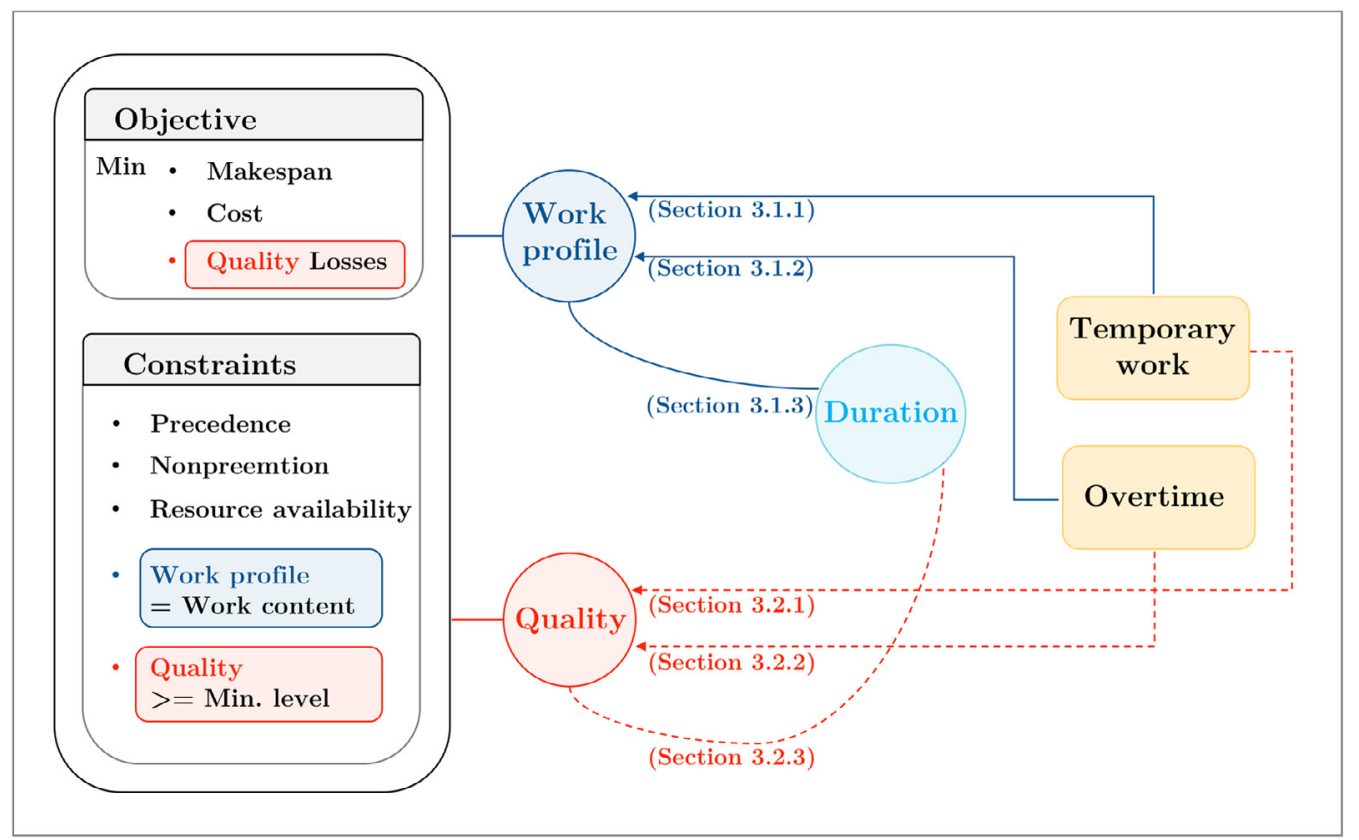

Fig. 1. The impact of additional manpower on quality and on processing times.

productivity with a variance in results which can be explained by the heterogeneity of projects and work types, site conditions, and definitions of overmanning. However, no shape of the relationship between productivity and overmanning was provided. To the best of our knowledge only Gunduz (2004) reported quantitative relationships from several past studies. These relationships can pessimistically be approximated by a linear function. Letting $\eta_{j t}$ be the variation rate of productivity, we have

$W_{j t}^{R}=\left(1-\eta_{j t}\right) \cdot\left(p_{j t}+u_{j t}\right) \quad \forall p_{j t}+u_{j t}>P_{j}^{\text {nor }}$,

where $W_{j t}^{R}$ is the number of workers in period $t$ who actually contribute to the execution of activity $j$. The linearity assumption between the productivity variation rate, $\eta_{j t}$, and the overmanning rate, $\left(\left(p_{j t}+u_{j t}\right) / P_{j}^{\text {nor }}\right)-1$, is written as

$\eta_{j t}=\gamma_{j} \cdot\left(\frac{p_{j t}+u_{j t}}{P_{j}^{\text {nor }}}-1\right)$,

where coefficient $\gamma_{j}>0$ measures the impact of overmanning on productivity losses. Replacing in Eq. (2) the rate $\eta_{j t}$ with its expression in Eq. (3), we obtain

$W_{j t}^{R} \equiv W_{j}^{R}\left(p_{j t}+u_{j t}\right)$

$$
=\left\{\begin{array}{l}
p_{j t}+u_{j t}, \quad \forall p_{j t}+u_{j t}=0, \ldots, P_{j}^{\text {nor }} \\
\left(1+\gamma_{j}\right) \cdot\left(p_{j t}+u_{j t}\right)-\left(\gamma_{j} / P_{j}^{\text {nor }}\right) \cdot\left(p_{j t}+u_{j t}\right)^{2}, \\
\forall p_{j t}+u_{j t}=P_{j}^{\text {nor }}+1, \ldots, P_{j}^{\max } .
\end{array}\right.
$$

Fig. 2 plots function $W_{j}^{R}\left(p_{j t}+u_{j t}\right)$ for several values of $\gamma_{j}$ and $P_{j}^{\text {nor }}=3$. When $\gamma_{j}=0$, overmanning has no impact on productivity. We have $W_{j}^{R}\left(p_{j t}+u_{j t}\right)=p_{j t}+u_{j t}$ which corresponds to a linear relationship between manpower and duration (doubling the workforce reduces by half the processing time).

When $\gamma_{j}>0$, we have a diminishing marginal productivity and function $W_{j}^{R}\left(p_{j t}+u_{j t}\right)$ reaches its maximum value for $p_{j t}+u_{j t}=\left(1+\gamma_{j}\right) P_{j}^{\text {nor }} / 2 \gamma_{j}$. This implies that beyond a number $\left\lfloor\left(1+\gamma_{j}\right) P_{j}^{\text {nor }} / 2 \gamma_{j}\right\rfloor$ of workers, adding a worker does not help to reduce the processing time. For instance, with $\gamma_{j}=0.50$, function $W_{j}^{R}\left(p_{j t}+u_{j t}\right)$ reaches its maximum for $\left\lfloor\left(1+\gamma_{j}\right) P_{j}^{\text {nor }} / 2 \gamma_{j}\right\rfloor=4$

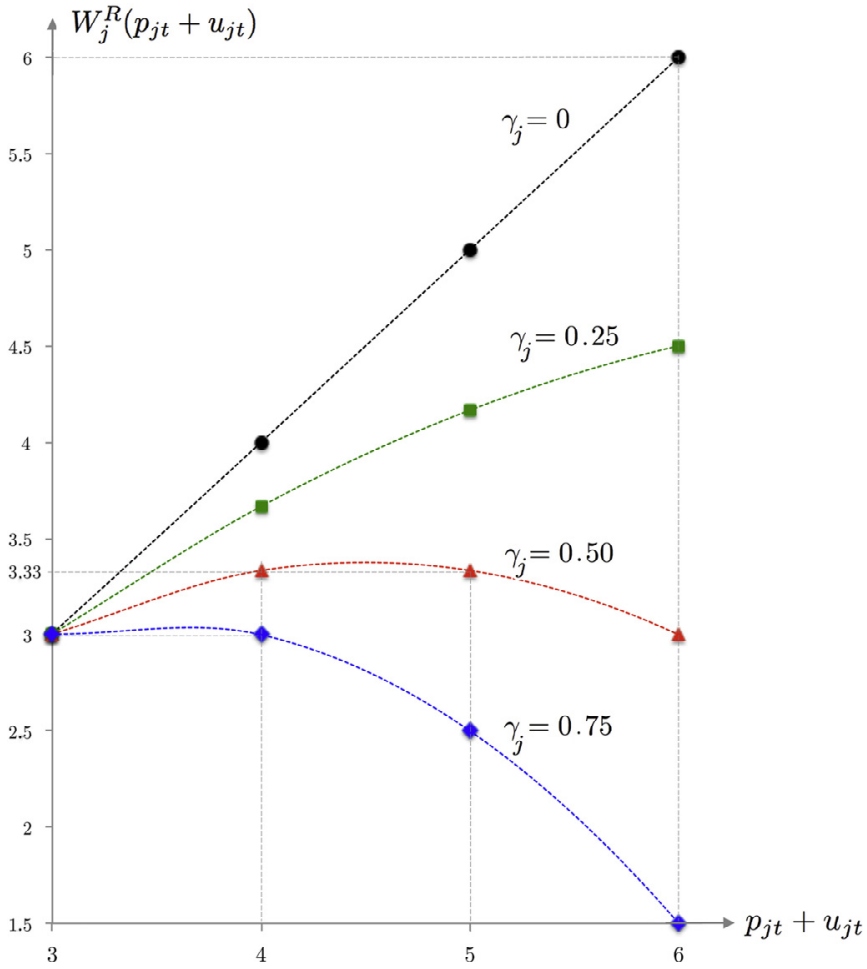

Fig. 2. Graph of function $W_{j}^{R}$ for several values of $\gamma_{j}$ and $P_{j}^{\text {nor }}=3$.

workers. We have $W_{j}^{R}(4)=W_{j}^{R}(5)=3.33$ meaning that 4 or 5 workers both provide the equivalent work of 3.33 workers who would actually make the activity progressing (see curve for $\gamma_{j}=0.50$ in Fig. 2).

Finally, there exists a value of $\gamma_{j}$ above which acceleration is precluded because the impact of overmanning on productivity is too strong. This value of $\gamma_{j}$ is obtained by solving $W_{j}^{R}\left(P_{j}^{\text {nor }}+\right.$ $1) \leq P_{j}^{\text {nor }}$ using Eq. (4), which gives $\gamma_{j} \geq P_{j}^{\text {nor }} /\left(P_{j}^{\text {nor }}+1\right)$ and for which we would set $P_{j}^{\max }=P_{j}^{\text {nor }}$. In our example, no acceleration is possible for $\gamma_{j} \geq 3 / 4$. 
To linearize function $W_{j}^{R}\left(p_{j t}+u_{j t}\right)$ given in Eq. (4) with possible values for $p_{j t}+u_{j t}$ in the set $\left\{0, \ldots, P_{j}^{\max }\right\}$, we note $y_{j t l}$ the binary variable that takes a value of 1 if $p_{j t}+u_{j t}$ takes a value of $l$ with $l \in\left\{0, \ldots, P_{j}^{\max }\right\}$ and 0 otherwise. Variables $p_{j t}+u_{j t}$ can therefore be written as

$p_{j t}+u_{j t}=\sum_{l=0}^{P_{j}^{\max }} l \cdot y_{j t l} \quad \forall j=1, \ldots, n, \quad t \in \mathcal{T}_{j}$,

with the following constraints that guarantee that the number of workers $p_{j t}+u_{j t}$ can take one and only one value

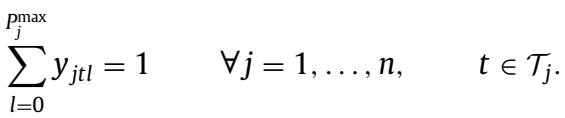

We therefore linearize function $W_{j t}^{R} \equiv W_{j}^{R}\left(p_{j t}+u_{j t}\right)$ as follows:

$W_{j t}^{R}=\sum_{l=0}^{P_{j}^{\max }} W_{j}^{R}(l) \cdot y_{j t l} \quad \forall j=1, \ldots, n, \quad t \in \mathcal{T}_{j}$,

where values of $W_{j}^{R}(l)$ are simply computed beforehand using Eq. (4).

\subsubsection{Effects of overtime on productivity}

Overtime is most commonly defined as the work performed over 8 hours a day and 40 hours a week (e.g. Hanna, Taylor, \& Sullivan, 2005). It has been well documented in the literature that overtime can adversely affect labor productivity due to fatigue, low morale and a phenomenon described by the U.S. Army where workers tend to pace themselves for longer days (U.S. Army Corps of Engineers, 1979). Rojas (2008) posits that 4 to 5 continuous weeks of overtime with 50 hours a week lead to a loss of labor efficiency of about $15 \%$ and can easily exceed $40 \%$ after 10 to 12 weeks. This finding is consistent with several studies of overtime on labour efficiency in various sectors and published by organizations such as the National Electrical Contractors Association (NECA) (1989), (Mechanical Contractors Association of America (MCAA) (2011), the Business Roundtable (BRT) (1980), or the Construction Industry Institute (Thomas \& Raynar, 1997). Alvanchi, Lee, and Abourizk (2012) also stress out that productivity losses may increase non-linearly with weeks of overtime because complete relief may not be achieved during the weekend.

Other studies advocate that overtime has no negative impact on productivity in specific contexts. Pencavel (2014) showed that below 49 weekly hours, variations in output are proportional to variations in hours, applying the least squares method to 122 weekly observations on munition workers. Circadian, a research and consulting company, stated that below 10 hours of overtime per week, the productivity is not affected for activities of maintenance and construction (Circadian, 2016). Beckers et al. (2004) conducted a survey-study among a representative sample of Dutch employees and did not find any association between fatigue and moderate overtime ( 1 to 8 hours a week). They even observed positive outcomes from overtime under favorable overtime working conditions such as voluntary overtime work, job variety, freedom to decide how to do the job and rewards (premium payment, recognition, promotions). These studies therefore suggest that below 8 to 10 overtime hours a week, productivity is not affected by overtime. As overtime regulation in Europe provides that full-time employees shall work overtime for a maximum of 8 hours a week, applying this regulation leads to getting the same productivity for overtime hours than for regular hours. We thus have

$W_{j t}^{O} \equiv W_{j}^{O}\left(h_{j t}\right)=h_{j t} / H_{R} \quad \forall j=1, \ldots, n, \quad t \in \mathcal{T}_{j}$.

Consequently, in our model, only overmanning has a negative impact on productivity.
Table 1

Additional manpower and processing time.

\begin{tabular}{llll}
\hline$d_{j}$ & \multicolumn{2}{l}{ Overmanning mainly } & Overtime only \\
\cline { 2 - 3 } & $\left(p_{j t}+u_{j t}-P_{j}^{\text {nor }}\right)_{t=1 . . d_{j}}$ & $\sum_{t=1}^{d_{j}} h_{j t} / H_{R}$ & $\sum_{t=1}^{d_{j}} h_{j t} / H_{R}$ \\
\hline 7 & $(0,0,0,0,1,1,1)$ & 0.3429 & 3 \\
6 & $(1,1,1,1,1,1)$ & 0.6856 & 6 \\
5 & $(2,2,2,2,2)$ & 0.4283 & 9 \\
\hline
\end{tabular}

\subsubsection{Processing time as a function of additional manpower and} illustration

The constraints on work content satisfaction in Eq. (1) can be rewritten using the expressions of $W_{j t}^{R} \equiv W_{j}^{R}\left(p_{j t}+u_{j t}\right)$ and $W_{j t}^{O} \equiv$ $W_{j}^{O}\left(h_{j t}\right)$ in Eq. (4) and Eq. (8), respectively

$$
\begin{aligned}
& \sum_{\left\{t \in \mathcal{T}_{j} \mid p_{j t}+u_{j t} \leq P_{j}^{\text {nor }}\right\}}\left(p_{j t}+u_{j t}\right)+\sum_{\left\{t \in \mathcal{T}_{j} \mid p_{j t}+u_{j t}>P_{j}^{\text {nor }}\right\}}\left(\left(1+\gamma_{j}\right) \cdot\left(p_{j t}+u_{j t}\right)\right. \\
& \left.-\left(\gamma_{j} / P_{j}^{\text {nor }}\right) \cdot\left(p_{j t}+u_{j t}\right)^{2}\right)+\sum_{t \in \mathcal{T}_{j}} h_{j t} / H_{R}=P_{j}^{\text {nor }} \cdot d_{j}^{\text {nor }} \\
& \quad \forall j=1, \ldots, n .
\end{aligned}
$$

With Eq. (9) we can now illustrate the relationship between duration and additional manpower. Let us consider an activity $j$ with $P_{j}^{\text {nor }}=3$ and $P_{j}^{\max }=5$ workers, $d_{j}^{\text {nor }}=8$ days, $H_{R}=8$ hours a day and an impact of overmanning on productivity $\gamma_{j}=0.0857$. In the normal mode, activity $j$ is processed by 3 workers a day over 8 days so the work content is 24 man-days. Table 1 provides for each reduced duration $d_{j}=7,6,5$ two types of manpower allocation, one with the highest number of regular workers (overmanning mainly) and one with overtime only.

As shown in Table 1 , to get a duration $d_{j}=7$ days using mainly workers in regular hours, one possible manpower allocation consists in adding to the three workers, one worker in overmanning per day over the last three days and to use a total of $0.3429 \times 8=2.7432$ overtime hours. Following Eq. (4), we have $W_{j}^{R}(3+1)=(1+0.0857) \times(3+1)-(0.0857 / 3) \times(3+$ $1)^{2}=3.8857$ which means that 4 workers are equivalent to 3.8857 efficient ones. Following Eq. (9), the work content is satisfied since $4 \times 3+3 \times W_{j}^{R}(4)+0.3429=12+3 \times 3.8857+0.3429=24$. Obviously, there is a multitude of manpower combinations to get $d_{j}=7$ and the optimal one will depend on workers' availabilities and unit costs of manpower. With overtime only (right side of Table 1), a duration $d_{j}=7$ days is reached with a total of $3 \times 8=24$ hours of overtime since we have $7 \times W_{j}^{R}(3)+3=7 \times 3+3=24$.

Fig. 3 plots the duration $d_{j}$ as a function of additional manpower $\sum_{t=1}^{d_{j}}\left(p_{j t}+u_{j t}-P_{j}^{\text {nor }}\right)+\sum_{t=1}^{d_{j}} h_{j t} / H_{R}$ for the two types of allocation displayed in Table 1 . As previously detailed, a duration $d_{j}=7$ days is obtained with the work of 3.3429 additional workers when overmanning is mainly used or with 24 overtime hours which corresponds to the daily work of 3 additional workers. Due to productivity losses when there is overmanning, the relationship between the duration of an activity and manpower is decreasing and convex, as illustrated in Fig. 3 (blue dotted curve, overmanning mainly). This relationship is linear when overtime is exclusively utilized (red solid line in Fig. 3) or if $\gamma_{j}=0$.

\subsection{Relationship between quality of an activity and additional manpower}

Quality of individual activities can be measured as a percentage of the checked items in their quality control checklist. The highest quality level $q_{j}^{\text {nor }}$ which is reached in normal mode usually equals one, meaning that $100 \%$ of the items are checked. We let parameter $\lambda_{j}^{R}$ denote the quality loss resulting from one day of regular 


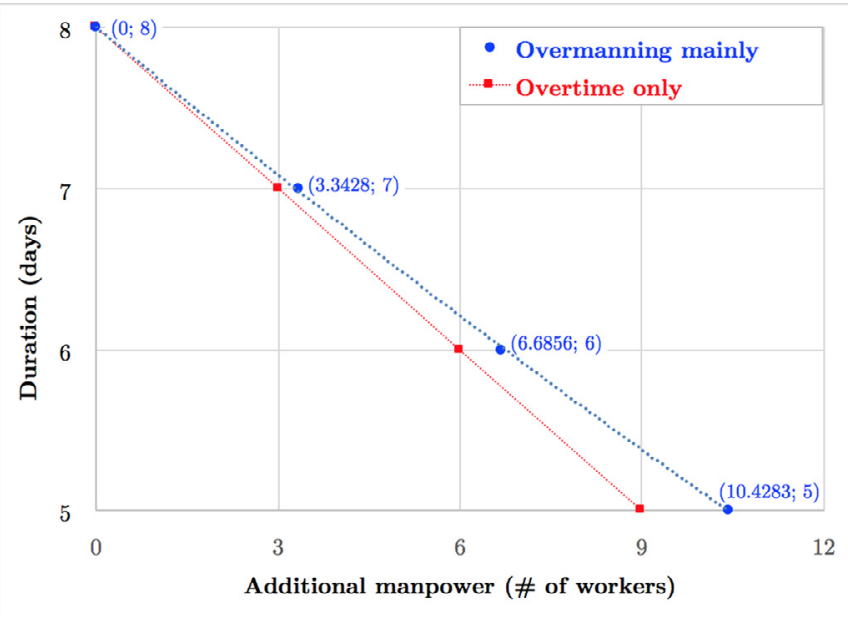

Fig. 3. Relationship between duration and manpower.

work ( $H_{R}$ hours) in situation of overmanning and parameter $\lambda_{j}^{O}$, the quality loss associated with one hour of overtime. For instance a value $\lambda_{j}^{R}=0.025$ means that one worker in situation of overmanning for $H_{R}$ hours (one day) induces on average the non-validation of $2.50 \%$ of the checklist items of activity $j$. A value $\lambda_{j}^{O}=0.00625$ expresses that one hour of overtime leads on average to the nonvalidation of $0.625 \%$ of the items in the checklist. Parameters $\lambda_{j}^{R}$ and $\lambda_{j}^{O}$ can also be interpreted as probabilities of deficiency occurrence (Kim et al., 2012). Letting $L_{j t}^{R} \equiv L_{j}^{R}\left(p_{j t}+u_{j t} ; \lambda_{j}^{R}\right)$ and $L_{j t}^{O} \equiv$ $L_{j}^{O}\left(h_{j t} ; \lambda_{j}^{O}\right)$ be respectively the quality losses associated with the use of overmanning and overtime on activity $j$ in period $t$, the individual quality $q_{j}$ can be written as

$q_{j}=q_{j}^{\text {nor }}-\sum_{t \in \mathcal{T}_{j}}\left(L_{j t}^{R}+L_{j t}^{O}\right)$.

While numerous studies have investigated the impact of overtime on quality, the effect of overmanning on quality remains scarcely explored. To the best of our knowledge, Li et al. (2000) are the only authors who quantified the negative impact of both overtime and additional resources on quality. Using data collected from building construction projects, they assessed the effect of different combinations of overtime and additional resources on the quality of these projects. Overtime and additional resources were expressed as percentages but the percentage bases were not reported. Consequently, this study is hardly exploitable to infer a relationship between quality of an activity and additional manpower. Nevertheless, it has at least the merit to show that the impact of overtime hours on quality is about twice the effect of overmanning hours

$\lambda_{j}^{O}=\rho \cdot \lambda_{j}^{R} / H_{R}, \quad \rho \simeq 2$.

In the remainder of this Section, we discuss the shapes of functions $L_{j}^{R}$ and $L_{j}^{O}$ to formulate a continuous and direct relationship between quality and manpower, whereas existing contributions focus solely on the relationship between quality and processing time (for a survey, see Orm \& Jeunet, 2018).

\subsubsection{Quality and overmanning}

As previously mentioned, the study of Li et al. (2000) does not allow to derive an analytical relationship between quality and overmanning. We thus relied upon the experience of Alstom's managers. They posit that a linear relationship between quality and overmanning can reasonably be assumed when overmanning is limited to a few additional workers per activity with dedicated workspaces. The quality decrease is therefore mainly due to a dilution of supervision and not to a site congestion. According to these managers, if workstations were not designed to accommodate extra workers then congestion would lead to quality losses exponentially increasing with overmanning (the relationship between overmanning and quality would be concave). But in most manufacturing industries there exists a buffer capacity for temporary purposes so we can reasonably assume that

$$
\begin{aligned}
L_{j t}^{R} & \equiv L_{j}^{R}\left(p_{j t}+u_{j t}\right) \\
& =\left\{\begin{array}{l}
0, \quad \forall p_{j t}+u_{j t}=0, \ldots, P_{j}^{\text {nor }} \\
\lambda_{j}^{R} \cdot\left(p_{j t}+u_{j t}-P_{j}^{\text {nor }}\right), \quad \forall p_{j t}+u_{j t}=P_{j}^{\text {nor }}+1, \ldots, P_{j}^{\max }
\end{array}\right.
\end{aligned}
$$

To linearize $L_{j}^{R}\left(p_{j t}+u_{j t}\right)$ we adopt a similar approach to the one we used for linearizing $W_{j}^{R}\left(p_{j t}+u_{j t}\right)$ in Eq. (7). We obtain

$L_{j t}^{R}=\sum_{l=0}^{P_{j}^{\max }} L_{j}^{R}(l) \cdot y_{j l t} \quad \forall j=1, \ldots, n, \quad t \in \mathcal{T}_{j}$,

with the same binary variables as in Eq. (5) and with values of quality losses $L_{j}^{R}(l)$ computed beforehand as follows:

$$
L_{j}^{R}(l)=\left\{\begin{array}{ll}
0, & \forall l=0, \ldots, P_{j}^{\text {nor }} \\
\lambda_{j}^{R} \cdot\left(l-P_{j}^{\text {nor }}\right), & \forall l=P_{j}^{\text {nor }}+1, \ldots, P_{j}^{\max }
\end{array} \quad \forall j=1, \ldots, n .\right.
$$

\subsubsection{Quality and overtime}

In the literature, quality is assumed to be a function of the processing time which in turn depends on the amount of resources. Zhang, Du, and Zhang (2014) formulate the quality of an activity as a quadratic function of its processing time for specific activities in construction like concreting and compaction. In Liberatore and Pollack-Johnson (2013), the quality of an activity is assumed to be a bivariate normal function of its cost and duration in new product development or when efficiencies of human resources are heterogeneous. Therefore, none of these non-linear relationships can be used to formulate the quality decrease due to acceleration with additional manpower in the manufacturing environment we consider. We thus assume that quality linearly decreases with duration, as in Babu and Suresh (1996) and Khang and Myint (1999) who consider a project expedited with overtime only. This amounts to assuming that quality linearly decreases with overtime, which is a reasonable assumption since the law significantly limits overtime. Furthermore, Alstom's quality managers supported this assumption. This is written as

$L_{j t}^{O}=\lambda_{j}^{O} \cdot h_{j t}$.

Proof. When there is no overmanning, activity $j$ uses a number $P_{j}^{\text {nor }} \cdot d_{j}$ of regular workers over its processing time $d_{j}$. This comes from the usual assumption that the number of permanent workers assigned to any activity in each period is at least equal to this number in the normal mode, $p_{j t} \geq P_{j}^{\text {nor }}$ (see for instance Naber \& Kolisch, 2014). Letting $S_{j}$ be the actual start time of activity $j$, the constraint on its work content (Eq. (9)) is written as $P_{j}^{\text {nor }} \cdot d_{j}+\sum_{t=S_{j}, \ldots, S_{j}+d_{j}}\left(h_{j t} / H_{R}\right)=P_{j}^{\text {nor }} \cdot d_{j}^{\text {nor }}$. This gives the volume of overtime hours to reduce the processing time from $d_{j}^{\text {nor }}$ to $d_{j}$, that is $\sum_{t=S_{j}, \ldots, S_{j}+d_{j}} h_{j t}=H_{R} \cdot P_{j}^{\text {nor }} \cdot d_{j}^{\text {nor }}-H_{R} \cdot P_{j}^{\text {nor }} \cdot d_{j}$. Besides, from Eq. (10), we have $q_{j}=q_{j}^{\text {nor }}-\lambda_{j}^{O} \cdot \sum_{t=S_{j}, \ldots, S_{j}+d_{j}} h_{j t}$. 
Table 2

Additional manpower and processing time.

\begin{tabular}{llllll}
\hline$d_{j}$ & \multicolumn{2}{l}{ Overmanning mainly } & & & Overtime only \\
\cline { 2 - 3 } \cline { 5 - 6 } & Add. workers & $q_{j}$ & & Add. workers & $q_{j}$ \\
\hline 7 & 3.3429 & 0.9079 & 3 & 0.85 \\
6 & 6.6856 & 0.8157 & 6 & 0.70 \\
5 & 10.4283 & 0.7286 & 9 & 0.55 \\
4 & 14.6281 & 0.6186 & 12 & 0.40 \\
3 & 20.5419 & 0.4729 & 15 & 0.25 \\
\hline
\end{tabular}

Substituting the sum of overtime hours for its expression into that of $q_{j}$, we get an affine function between $q_{j}$ and $d_{j}$

$$
\begin{gathered}
q_{j}=\left(\lambda_{j}^{O} \cdot H_{R} \cdot P_{j}^{\text {nor }}\right) d_{j}+\left(q_{j}^{\text {nor }}-\lambda_{j}^{O} \cdot H_{R} \cdot P_{j}^{\text {nor }} \cdot d_{j}^{\text {nor }}\right), \\
\forall p_{j t}+u_{j t}=P_{j}^{\text {nor }}, \quad t=S_{j}, \ldots, S_{j}+d_{j} .
\end{gathered}
$$

3.2.3. Relationships between quality, additional manpower, and processing time and numerical illustration

Following our assumptions on functions $L_{j}^{R}$ and $L_{j}^{O}$, we can provide a new expression for quality $q_{j}$, using Eqs. (12) and (15)

$q_{j}=q_{j}^{\text {nor }}-\lambda_{j}^{R} \sum_{\left\{t \in \mathcal{T}_{j} \mid p_{j t}+u_{j t}>P_{j}^{\text {nor }}\right\}}\left(p_{j t}+u_{j t}-P_{j}^{\text {nor }}\right)-\lambda_{j}^{O} \sum_{t \in \mathcal{T}_{j}} h_{j t}$.

Let us now study the relationship between quality and duration. This relationship is linear when only overtime is used, as shown by Eq. (16). When overmanning is combined with overtime, an analytical relationship between the quality $q_{j}$ and the processing time $d_{j}$ can hardly be derived for there exists several allocations of additional workers over processing periods leading to a same duration $d_{j}$ with different impacts on quality. However, under some assumptions, we can first derive a relationship between overmanning and duration and next between quality and duration. With no overtime and if we assume a constant number of workers in overmanning over the $d_{j}$ processing periods, $p_{j t}+u_{j t}=p_{j}+u_{j}, \forall t=S_{j}, \ldots, S_{j}+d_{j}$, the constraint on the work content is written as $d_{j} \cdot W_{j}^{R}\left(p_{j}+u_{j}\right)=P_{j}^{\text {nor }} \cdot d_{j}^{\text {nor }}$. Replacing $W_{j}^{R}\left(p_{j}+u_{j}\right)$ with its expression (see Eq. (4)), we get $d_{j} \cdot\left(\left(1+\gamma_{j}\right)\right.$. $\left.\left(p_{j}+u_{j}\right)-\left(\gamma_{j} / P_{j}^{\text {nor }}\right) \cdot\left(p_{j}+u_{j}\right)^{2}\right)-P_{j}^{\text {nor }} \cdot d_{j}^{\text {nor }}=0$. This quadratic equation has the following acceptable root which provides a non linear relationship between overmanning and duration

$p_{j}+u_{j}=\left(P_{j}^{\text {nor }} / 2 \gamma_{j}\right) \cdot\left(\left(1+\gamma_{j}\right)-\frac{1}{d_{j}} \sqrt{\left(1+\gamma_{j}\right)^{2} \cdot d_{j}^{2}-4 \gamma_{j} d_{j}^{\text {nor }} \cdot d_{j}}\right)$.

Besides, Eq. (17) gives $q_{j}=q_{j}^{\text {nor }}-\lambda_{j}^{R} d_{j}\left(p_{j}+u_{j}-P_{j}^{\text {nor }}\right)$ in which we replace $p_{j}+u_{j}$ with its expression in Eq. (18). We finally obtain $q_{j}$ as a non linear function of $d_{j}$

$$
\begin{aligned}
& q_{j}= q_{j}^{\text {nor }}-\lambda_{j}^{R} \cdot\left(P_{j}^{\text {nor }} / 2 \gamma_{j}\right) \\
& \times\left(\left(1-\gamma_{j}\right) \cdot d_{j}-\sqrt{\left(1+\gamma_{j}\right)^{2} \cdot d_{j}^{2}-4 \gamma_{j} d_{j}^{\text {nor }} \cdot d_{j}}\right), \\
& \forall p_{j t}+u_{j t}=p_{j}+u_{j}>P_{j}^{\text {nor }}, \quad t=S_{j}, \ldots, S_{j}+d_{j} .
\end{aligned}
$$

Obviously, $p_{j}+u_{j}$ in Eq. (18) has little chance to be an integer, in which case we can round its value and complete with overtime to meet the work content. To illustrate, let us consider our example in Section 3.1 .3 with parameters values $q_{j}^{\text {nor }}=1, \lambda_{j}^{R}=$ 0.025 and $\lambda_{j}^{O}=0.00625$. Table 2 provides the values of quality $q_{j}$ for the same manpower allocations as those in Table 1 . We extended the computations to lower values of duration, $d_{j}=4,3$,

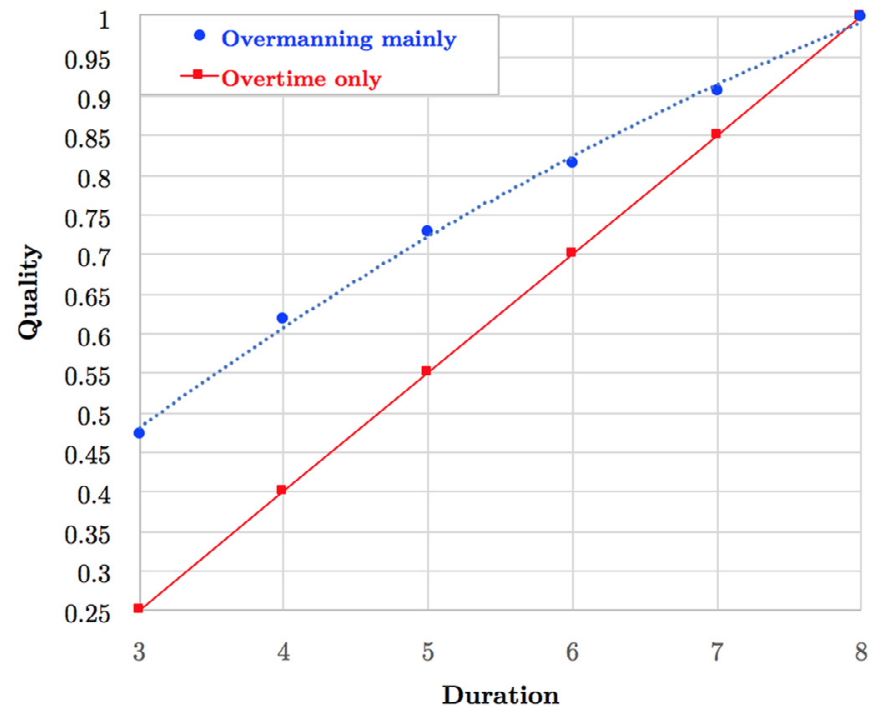

Fig. 4. Relationship between quality and duration.

in order to better emphasize the concavity of the relationship between quality and duration when overmanning is mainly utilized. In column "Add. workers", we report the sum $\sum_{t=1}^{d_{j}}\left(p_{j t}+\right.$ $\left.u_{j t}-P_{j}^{\text {nor }}+h_{j t} / H_{R}\right)$. For instance, for a reduced duration $d_{j}=7$ obtained with overmanning mainly with $\left(p_{j t}+u_{j t}-P_{j}^{\text {nor }}\right)_{t=1 . .7}=$ $(0,0,0,0,1,1,1)$, this sum equals 3.3429 which corresponds to one additional worker for 3 days and a total of $0.3429 \times 8=$ 2.7432 overtime hours. Using Eq. (17), we have $q_{j}=1-3 \lambda_{j}^{R}-$ $2.7432 \lambda_{j}^{O}=0.9079$. Let us note that a duration $d_{j}=7$ can also be obtained with $\left(p_{j t}+u_{j t}-P_{j}^{\text {nor }}\right)_{t=1 . .7}=(0,0,0,0,0,2,1)$, which has a work content of 23.6001, and overtime hours equal to $(24-23.6001) \times 8=3.1992$. The corresponding quality is lower, with $q_{j}=1-3 \lambda_{j}^{R}-3.1992 \lambda_{j}^{O}=0.9050$. But such an allocation of additional manpower can be optimal if no additional worker is available on the fifth day. With overtime only, duration $d_{j}=7$ is obtained by utilizing $3 \times 8=24$ overtime hours and a quality $q_{j}=1-24 \lambda_{j}^{O}=0.85$.

To reach a duration $d_{j}=4$ with overmanning mainly, Eq. (18) gives $p_{j}+u_{j}=6.71$. We therefore set $p_{j t}+u_{j t}=7$ in the first 2 days and $p_{j t}+u_{j t}=6$ in the last 2 days. The associated work content is equal to 23.3719 , so we add $(24-23.3719) \times 8=5.0248$ overtime hours. The corresponding quality is $q_{j}=1-14 \times 0.025-5.0248 \times 0.00625=0.6186$. For a duration $d_{j}=3$, Eq. (18) provides $p_{j}+u_{j}=9.99$. The manpower allocation $(10,10,9)$ has a work content equal to 23.4581 so $(24-23.4581) \times 8=4.3352$ overtime hours are utilized. The quality $q_{j}$ drops to 0.4729 .

Fig. 4 plots the quality $q_{j}$ as a function of the duration $d_{j}$ for the two types of allocation displayed in Table 2 . When overmanning is mainly used, the relationship between $q_{j}$ and $d_{j}$ is concave as illustrated in this Figure.

\subsection{Optimization program}

One method for solving multi-objective optimization problems is the lexicographic approach which requires an ordinal ranking of the achievement functions. The approach proceeds iteratively, solving first the most important objective as a single-objective problem. The second objective is then solved in which the optimal solution obtained in the previous step is added as a new constraint. The process is repeated until the whole sequence of single-objective optimization problems is solved Stadler (1988) for 
a reference book and Russell and Taghipour (2019) for a recent application in scheduling).

This optimization approach is consistent with our planning problem where the objective functions of makespan $C_{\max }$, total cost $B$, and overall quality losses $L$ are ranked in this order of importance to the decision maker. The method therefore consists of solving sequentially the following single-objective optimization problems: (1) $\min C_{\max }$; (2) $\min B$ subject to $C_{\max } \leq C_{\max }^{*}$ where $C_{\max }^{*}$ is the optimal value of (1); (3) $\min L$ subject to $C_{\max } \leq C_{\max }^{*}$ and $B \leq B^{*}$ where $B^{*}$ is the optimal value of (2).

\subsubsection{Preprocessing}

The bounds of the set $\mathcal{T}_{j}=\left\{E S_{j}, \ldots, L F_{j}\right\}$ are computed as follows. The earliest start time $E S_{j}$ is defined as $E S_{j}=$ $\max _{i \in \Gamma^{-1}(j)}\left\{E S_{i}+\underline{d}_{i}\right\}$ where $\underline{d}_{j}$ is a lower bound on the processing time. This bound is obtained by hypothetically allocating the maximum of additional manpower to activity $j$, that is $\underline{d}_{j}=\left\lceil P_{j}^{\text {nor }}\right.$. $\left.d_{j}^{\text {nor }} /\left(W_{j}^{R}\left(P_{j}^{\max }\right)+\left(H_{O} / H_{R}\right) \cdot P_{j}^{\text {nor }}\right)\right\rceil$. We set $E S_{0}=0$ and the earliest start time of dummy activity $n+1$ (end of the project) is a lower bound $\underline{C}_{\max }$ on the project completion time, $\underline{C}_{\max }=E S_{n+1}$.

The latest finish time $L F_{j}$ is based upon normal processing times. We first compute the earliest start time in the normal mode $E S_{j}^{\text {nor }}=\max _{i \in \Gamma^{-1}(j)}\left\{E S_{i}^{\text {nor }}+d_{i}^{\text {nor }}\right\}$ with $E S_{0}^{\text {nor }}=0$. The latest start time is then determined, with $L S_{j}^{\text {nor }}=\min _{i \in \Gamma(j)}\left\{L S_{i}^{\text {nor }}\right\}-d_{j}^{\text {nor }}$ and $L S_{n+1}^{\text {nor }}=E S_{n+1}^{\text {nor }}$. We finally obtain $L F_{j}=L S_{j}^{\text {nor }}+d_{j}^{\text {nor }}$. The latest finish time of activity $n+1$ simply corresponds to the project completion time in the normal mode, $C_{\max }^{\text {nor }}$, and constitutes an upper bound to the makespan $C_{\max }$. We have $C_{\max }^{\text {nor }}=L F_{n+1}$.

The total number of permanent workers with skill $k$ dedicated to the project must allow for its execution in the normal mode and is thus computed as

$\mathcal{P}_{k}=\max _{t=1, \ldots, C_{\max }^{\text {nor }}}\left(\sum_{\left\{j \in V_{k} \mid t \in\left\{E S_{j}^{\text {nor }}, \ldots, E S_{j}^{\text {nor }}+d_{j}^{\text {nor }}\right\}\right\}} P_{j}^{\text {nor }}\right), \quad \forall k=1, \ldots, K$.

\subsubsection{Formulation}

The first objective is to minimize the project completion time $C_{\max }$ with

$C_{\max } \geq t \cdot z_{j t} \quad \forall j=1, \ldots, n, \quad t \in \mathcal{T}_{j}$,

and

$\underline{C}_{\max } \leq C_{\max } \leq C_{\max }^{\text {nor }}$

Without loss of generality, we assume that the cost of one hour of permanent work, $c_{P}$, is identical for all skills. The same holds for the cost $c_{U}$ of one hour of temporary work and the cost $c_{O}$ of one hour of overtime. The second objective to be minimized under the optimal project duration constraint is the total cost $B$ defined as the sum of the cost of permanent work, temporary work and overtime

$B=\sum_{j=1}^{n} \sum_{t \in \mathcal{T}_{j}}\left(c_{P} \cdot H_{R} \cdot p_{j t}+c_{U} \cdot H_{R} \cdot u_{j t}+c_{O} \cdot h_{j t}\right)$.

The third objective is to minimize the project overall quality losses $L$ under optimal duration and cost of the project, with

$L=\sum_{j=1}^{n}\left(\sum_{l=0}^{P_{j}^{\max }} L_{j}^{R}(l) \cdot y_{j l t}+\lambda_{j}^{O} \sum_{t \in \mathcal{T}_{j}} h_{j t}\right)$,

and values of quality losses $L_{j}^{R}(l)$ computed beforehand using Eq. (14).
Precedence constraints state that activities can not start before completion of their predecessor activities

$\sum_{\tau=E S_{j}}^{t} z_{j \tau} \leq d_{j}^{\text {nor }} \cdot\left(1-z_{i t}\right) \quad \forall j=1, \ldots, n, \quad i \in \Gamma^{-1}(j), \quad t \in \mathcal{T}_{i} \cap \mathcal{T}_{j}$.

Nonpreemption constraints ensure that activities execution can not be interrupted and restarted later. They apply to any activity $j$ with a normal duration $d_{j}^{\text {nor }} \geq 2$ and such that $L F_{j}-E S_{j} \geq 3$ otherwise interruption can never occur

$$
\begin{aligned}
& \sum_{\tau=t+1}^{L F_{j}} z_{j \tau}+d_{j}^{\text {nor }}\left(z_{j t}-z_{j t+1}\right) \leq d_{j}^{\text {nor }} \\
& \forall j=1, \ldots, n, \quad \text { with } d_{j}^{\text {nor }} \geq 2, \\
& \forall t=E S_{j}, \ldots, L F_{j}-3 \quad \text { with } L F_{j}-E S_{j} \geq 3 .
\end{aligned}
$$

Workforce requirement. The number of workers allocated to any activity $j$ over its processing periods must satisfy its work content

$\sum_{t \in \mathcal{T}_{j}}\left(\sum_{l=0}^{P_{j}^{\max }} W_{j}^{R}(l) \cdot y_{j t l}+h_{j t} / H_{R}\right)=P_{j}^{\text {nor }} \cdot d_{j}^{\text {nor }} \quad \forall j=1, \ldots, n$,

where values of $W_{j}^{R}(l)$ are computed beforehand using Eq. (4) and $h_{j t} / H_{R}$ is the equivalent number of regular workers corresponding to the volume of overtime hours. Constraints on binary variables $y_{j t l}$ in Eq. (5) and (6) are added to the optimization program.

Resources availability. For each skill $k$ the number of permanent workers allocated to the execution of activities requiring that skill in each period should not exceed the total permanent workforce with skill $k$ dedicated to the project

$\sum_{j \in V_{k}} p_{j t} \leq \mathcal{P}_{k} \quad \forall k=1, \ldots, K, \quad t \in \bigcup_{j \in V_{k}} \mathcal{T}_{j}$

As in Heimerl and Kolisch (2010), we assume that only permanent workers can work overtime. Recalling that in each period the number of overtime hours per worker is limited to $H_{O}$ hours, the following constraints guarantee that there is enough permanent workers in each period to ensure overtime hours

$\sum_{j \in V_{k}} h_{j t} \leq H_{O} \sum_{j \in V_{k}} p_{j t} \quad \forall k=1, \ldots, K, \quad t \in \bigcup_{j \in V_{k}} \mathcal{T}_{j}$

Summation over activities in constraints (28) means that some permanent workers with skill $k$ allocated in regular time to a given activity may work overtime on a different activity also requiring skill $k$.

Due to legal constraints, each permanent worker can not work more than $H_{O W}$ hours overtime per week (5 days) which is written as

$\sum_{\tau=t}^{t+4}\left(\sum_{\left\{j \in V_{k} \mid \tau \in \mathcal{T}_{j}\right\}} h_{j \tau}\right) \leq(1 / 5) \cdot H_{O W} \cdot \sum_{\tau=t}^{t+4}\left(\sum_{j \in\left\{j \in V_{k} \mid \tau \in \mathcal{T}_{j}\right\}} p_{j \tau}\right)$,

$\forall k=1, \ldots, K, \quad t=\min _{j \in V_{k}} E S_{j}, \ldots, \max _{j \in V_{k}} L F_{j}-4$.

Constraints (29) consider the total amount of overtime hours (right hand side) performed by the average number of permanent workers active on each day of the week. Our model, as a planning model, does not consider each permanent worker individually as this should be the case for an operational model of allocating individuals to the execution of activities. 
Organizational constraints. We assume that the number of permanent workers assigned to any activity in each period is at least equal to this number in the normal mode

$p_{j t} \geq P_{j}^{\text {nor }} \cdot z_{j t} \quad \forall j=1, \ldots, n, \quad t \in \mathcal{T}_{j}$.

This is a standard assumption (see for instance Naber \& Kolisch, 2014). Besides, constraints (30) avoid situations where only temporary workers would be dedicated to the execution of an activity.

No more than $P_{j}^{\max }$ workers in regular working hours can be allocated to the execution of activity $j$ in any period

$p_{j t}+u_{j t} \leq P_{j}^{\max } \cdot z_{j t} \quad \forall j=1, \ldots, n, \quad t \in \mathcal{T}_{j}$.

In each processing period, workers overtime are never in an overmanning situation, which implies that their maximum number equals $P_{j}^{\text {nor }}$ so that overtime hours can not exceed $P_{j}^{\text {nor }} \cdot H_{O}$

$h_{j t} \leq P_{j}^{\text {nor }} \cdot H_{O} \cdot z_{j t} \quad \forall j=1, \ldots, n, \quad t \in \mathcal{T}_{j}$.

Constraints (32) also ensure that if there is no worker during regular hours $\left(z_{j t}=0\right)$ then overtime can not take place $\left(h_{j t}=0\right)$. Let us note that overtime hours can be performed by workers other than those who were dedicated to the activity during regular hours.

Quality constraints. When activity $j$ is accelerated its quality $q_{j}$ degrades with overmanning and overtime usage but must not fall below a threshold $q_{j}^{\min }$ corresponding to the rate of unacceptable flaws in the checklist. This is written as $q_{j}^{\text {nor }}-\sum_{t \in \mathcal{T}_{j}}\left(L_{j t}^{R}+L_{j t}^{O}\right) \geq$ $q_{j}^{\min }$. Using the linearization, we have

$q_{j}^{\text {nor }}-\lambda_{j}^{O} \sum_{t \in \mathcal{T}_{j}} h_{j t}-\sum_{t \in \mathcal{T}_{j}} \sum_{l=0}^{P_{j}^{\max }} L_{j}^{R}(l) \cdot y_{j l t} \geq q_{j}^{\min } \quad \forall j=1, \ldots, n$,

where values of $L_{j}^{R}(l)$ are computed beforehand according to Eq. (14).

It should be noted that our formulation is partly inspired by the first of the four MIP Models for resource-constrained project scheduling with flexible resource profiles (namely FP-DT1) developed by Naber and Kolisch (2014). We thus used $z_{j t}$ to indicate the processing status of activity $j$ in period $t$ and this choice was motivated by the intuitive definition of such variables. Similarities between model FP-DT1 and our formulation are however limited to the objective of makespan minimization in Eq. (20) and precedence and nonpreemption constraints in Eq. (24) and Eq. (25), respectively.

\section{Numerical results}

We considered several projects taken from the literature as well as a case study of the production of a motor coach at Alstom. We first describe the instances and the experimental design that involves 2 phases (Section 4.1). In the first phase, our incentive is to show the ability of our MILP formulation to solve the instances of the case study generated in a full factorial design. With numerous instances, we can also provide an analysis of the trade-off between duration, cost and quality (Section 4.2). The second phase aims at assessing the efficiency of our MILP formulation when applied to other projects with specific combinations of parameters values defining easy and hard instances (Section 4.3).

\subsection{Project types, parameters setting and experimental design}

Project types. We considered 7 manufacturing projects. The first one is a case study of the production of a very high speed train motor coach at Alstom, a world leader in integrated transport systems and headquartered in France. ${ }^{1}$ The data were collected from the production plant in Belfort, France. The project includes 36 activities with 18 crashable ones (normal duration greater than one day and allowed quality losses). This case study was then extended by rendering the 36 activities all crashable. Next we added 4 activities to get a project with 40 crashable activities.

The other projects come from the literature. We considered a robot type palletizing system installation with 18 activities (Kim, Kang, \& Hwang, 2012). A typical construction project of a two-story building with 18 activities was taken from Mohammadipour and Sadjadi (2016). Finally, we took 2 instances from PSPLIB (Kolisch \& Sprecher, 1996) with 30 activities and an average number of successors per activity of 1.5 and 2.125 respectively.

Skills, number of workers and normal processing times

The production of the train motor coach requires $K=7$ skills (welding; painting; assembly; wiring; electricity; pneumatic; electronic automation). Any activity is processed on a single workstation and each workstation hosts workers with a single skill only (workstations do not mix skills). Alstom provided for each activity $j$, the number of workers $P_{j}^{\text {nor }}$; the maximum number of workers $P_{j}^{\max }$ and the normal processing time $d_{j}^{\text {nor }}$.

For the other projects, specialities $k=1 . . K$ and number of permanent workers $P_{j}^{\text {nor }}, P_{j}^{\max }$ were set to consistent values with respect to the number of activities, their description if any, and their processing times. $^{2}$

Minimum level of individual quality. Minimum quality levels can be derived from the number of unacceptable flaws in quality control inspection checklists. For our case study, we could obtain these checklists. An example of such a list is provided for the activity of the motor coach painting $(j=6)$ in Table 6 in Appendix B. The checklist contains 9 items among which 3 minor defects do not need rework (item 3: small localized painting defect; item 7: invisible paint peel-off; item 8 : light scratches paint). We thus set $q_{6}^{\min }=2 / 3$. Testing activities such as pneumatic testing or safety equipments testing can not be accelerated: those with a normal duration $d_{j}^{\text {nor }}=1$ and those for which not a single flaw is acceptable, that is $q_{j}^{\min }=q_{j}^{\text {nor }}=1$. These activities are testing activities.

For the robotic palletizing system, we took the minimum quality levels $q_{j}^{\min }$ as evaluated by the authors (Kim et al., 2012). For the other projects, three minimum quality levels $(0.95,0.80$ and 0.67$)$ were randomly assigned to each activity.

Costs and working time. Using the informations provided by Alstom, we set for all projects $c_{P}=22.52$ euros; $c_{U}=1.20 c_{P}$ and $c_{O}=$ $1.25 c_{p}$. In accordance with French law, we have for each worker $H_{R}=7.8$ regular hours a day, $H_{O}=4$ overtime hours a day and $H_{O W}=8$ hours a week.

Impact of overmanning on productivity $\left(\gamma_{j}\right)$ and unit quality losses $\left(\lambda_{j}^{R}, \lambda_{j}^{O}\right)$. Observations of processing times and quality levels in specific conditions of overmanning and overtime utilization would allow for an estimation of parameters $\gamma_{j}, \lambda_{j}^{R}$ and $\lambda_{j}^{O}$ as explained in Appendix C. Unfortunately, Alstom does not record such data and to the best of our knowledge, there is no empirical study that quantifies such impacts. Consequently, we adopted a simulation approach. In order to set consistent values for these parameters and to account for a wide variety of situations, we developed the following reasoning. In Section 3.1.1, we have shown that function $W_{j}^{R}\left(p_{j t}+u_{j t}\right)$ reaches its maximum value for $p_{j t}+u_{j t}=$ $\left(1+\gamma_{j}\right) P_{j}^{\text {nor }} / 2 \gamma_{j}$. Thus, to allow for a maximum number of workers

\footnotetext{
1 https://www.alstom.com/company.

2 Data are available from the corresponding author upon request.
} 
Table 3

\begin{tabular}{|c|c|c|c|c|c|c|c|}
\hline Project description & $\begin{array}{l}\text { Robot type } \\
\text { palletizing }\end{array}$ & Random, PSPLIB & $\begin{array}{l}\text { Alstom } 18 \\
\text { crashable } \\
\text { activities }\end{array}$ & Random, PSPLIB & Two-story building & $\begin{array}{l}\text { Alstom } 36 \\
\text { crashable } \\
\text { activities }\end{array}$ & $\begin{array}{l}\text { Alstom } 40 \\
\text { crashable } \\
\text { activities }\end{array}$ \\
\hline Reference & $\begin{array}{l}\text { Kim et al. } \\
\text { (2012) }\end{array}$ & $\begin{array}{l}\text { Kolisch and } \\
\text { Sprecher (1996) }\end{array}$ & This paper & $\begin{array}{l}\text { Kolisch and } \\
\text { Sprecher (1996) }\end{array}$ & $\begin{array}{l}\text { Mohammadipour } \\
\text { and Sadjadi (2016) }\end{array}$ & This paper & This paper \\
\hline \# Activities & 18 & 30 & 36 & 30 & 18 & 36 & 40 \\
\hline Avg \# succ. & 1.105 & 1.5 & 10.26 & 2.125 & 1.3 & 1.026 & 1.0124 \\
\hline Duration(days) & 27 & 37 & 107 & 54 & 121 & 119 & 131 \\
\hline \#Variables & 1155 & 4882 & 5553 & 6143 & 6483 & 8872 & 10193 \\
\hline \#Constraints & 1366 & 4149 & 6241 & 5764 & 6984 & 9144 & 10533 \\
\hline Phase 1 (all param. values) & & & $\times$ & & & & \\
\hline Phase 2 (easy and hard) & $\times$ & $x$ & & $\times$ & $\times$ & $\times$ & $\times$ \\
\hline
\end{tabular}

in regular hours $P_{j}^{\max }$ in each processing period, parameter $\gamma_{j}$ must be such that $\gamma_{j} \leq P_{j}^{\text {nor }} /\left(2 P_{j}^{\max }-P_{j}^{\text {nor }}\right)$. We thus set $\gamma_{j} \equiv \gamma_{j}(\psi)=$ $\psi \cdot P_{j}^{\text {nor }} /\left(2 P_{j}^{\max }-P_{j}^{\text {nor }}\right)$, with $\psi \in\{0.05,0.20,0.40,0.60,0.80,1.00\}$ to represent an extensive range of productivity losses scenarios, starting with a negligible impact of overmanning on productivity and ending with a maximum impact.

It is reasonable to assume that companies limit the maximum number of workers on any activity so as to avoid rework. Thus, with $P_{j}^{\max }$ workers allocated to activity $j$ over its whole processing time $d_{j}$, the quality should not drop below $q_{j}^{\text {min }}$. We have $d_{j} \equiv d_{j}\left(P_{j}^{\max }\right)=\left(P_{j}^{\text {nor }} \cdot d_{j}^{\text {nor }}\right) / W_{j}^{R}\left(P_{j}^{\max }\right)$, where $W_{j}^{R}\left(P_{j}^{\max }\right)$ is computed using Eq. (4) with $\gamma_{j} \equiv \gamma_{j}(\psi)$. The corresponding quality $q_{j}$ is $q_{j}=q_{j}^{\text {nor }}-\lambda \lambda_{j}^{R} \cdot d_{j}\left(P_{j}^{\max }\right) \cdot\left(P_{j}^{\max }-P_{j}^{\text {nor }}\right)$ and imposing $q_{j} \geq q_{j}^{\min }$ leads to $\lambda_{j}^{R} \leq\left(q_{j}^{\text {nor }}-q_{j}^{\min }\right) /\left(d_{j}\left(P_{j}^{\max }\right) \cdot\left(P_{j}^{\max }-P_{j}^{\text {nor }}\right)\right)$. We thus set $\lambda_{j}^{R} \equiv \lambda_{j}^{R}(\varphi)=\varphi \cdot\left(q_{j}^{\text {nor }}-q_{j}^{\min }\right) /\left(d_{j}\left(P_{j}^{\max }\right) \cdot\left(P_{j}^{\max }-P_{j}^{\text {nor }}\right)\right)$, with parameter $\varphi$ in the same set of values as parameter $\psi$. In this way, we examine a wide range of scenarios where for example overmanning can strongly impact the productivity with a slight effect on quality (workers may work slowly while delivering high quality work) and conversely, workers may work fast (low value of $\psi$ ) with a low quality outcome (high value of $\varphi$ ).

As stated by Li et al. (2000), the negative impact on quality of overtime hours is about twice that of overmanning hours (see Eq. (11)). Managers of Alstom confirmed this order of magnitude but were unable to provide a sharper estimate. We thus adopted again a simulation approach, with $\lambda_{j}^{O} \equiv \lambda_{j}^{O}(\rho)=\rho \cdot \lambda_{j}^{R}(\varphi) / H_{R}$ and $\rho \in\{1.8,1.9,2.0,2.1,2.2\}$.

Experimental design. Our MILP was coded in C and linked with the CPLEX callable optimization library version 12.5. The experiments were executed on an Intel Xeon E5-2430 with a 2.20 gigahertz ( 16 Cores) CPU and 30 gigabytes RAM running the GNU/Linux Debian 7 operating system.

The experiment was divided into 2 phases. In the first phase, we considered the original case study with 36 activities and 18 crashable ones, with all combinations of parameters values. We thus solved 180 instances in order to analyze the trade-off between duration, cost and quality. We set a runtime limit for CPLEX to 3600 seconds throughout, for each of the three objectives to optimize consecutively. From the results we identified specific combinations of parameters values $\psi$ and $\varphi$ for which the solution time was minimum (easy instances) and maximum (hard instances). Thus, in the second phase, our intent is to study the efficiency of the MILP formulation over easy and hard instances for the 6 additional projects. For easy instances, we kept a time limit of 3600 seconds for each subproblem. For hard instances, the time limit was increased to 7200 seconds. Beyond a certain number of variables, we provided CPLEX with a starting solution. Table 3 summarizes the projects and experiment.
4.2. First phase: analysis of the trade-off between duration, cost and quality in our case study

Table 7 in Appendix B displays the data provided by Alstom for each activity $j=1 . .36$ of the project: description; set $\Gamma^{-1}(j)$ of predecessor activities; skill $k$; normal duration $d_{j}^{\text {nor }}$; and minimum quality level $q_{j}^{\mathrm{min}}$. Applying all combinations of parameters values to the project, we obtained $6 \times 6 \times 5=180$ instances, solving for each of them 3 optimization programs (lexicographic approach).

We obtained high quality solutions with optimal durations in all cases, cost values within $0.01 \%$ of optimum (average gap to best bound equals $0.006 \%$ ) and all quality values but four within $0.1 \%$ of optimal (the average gap to best bound equals $1.39 \%$ over these 4 cases, and $0.08 \%$ over all instances). It took on average 3 minutes in real time to solve one instance.

Results for the 180 instances are reported in Tables 8 and 9 in Appendix D where the first four columns describe the instance (instance number, impact of overmanning on productivity $\psi$, impact of overmanning on quality $\varphi$ and factor $\rho$ of the impact on quality of overtime compared with overmanning). The next three columns provide the duration reduction, cost increase and quality loss compared with the normal execution mode. In the normal mode, the project makespan is 107 days, with a labor cost of 60952.63 euros and a quality of 1 . The labor cost is the cost of 347 days of permanent work of all specialities at a unit cost $c_{p}=22.52$ euros per hour, with 7.8 regular hours a day $(347 \times 7.8 \times 22.52=60952.63$ euros).

Considering for example instance \#1 in Table 8 , the project makespan reaches its highest reduction of $16.82 \%$ (18 days) at the expense of a cost increase of $2.74 \%$ and a quality loss of $0.43 \%$. The last three columns provide the savings of permanent workers in hours due to the project acceleration compared with the 347 days of permanent work required in the normal mode as well as the number of temporary and overtime hours. Thus, in instance \#1, the project is completed using 313 days of permanent work instead of 347 which represents a saving in permanent hours of $34 \times 7.8=265.2$ with a use of 23 days of temporary work that is $23 \times 7.8=179.4$ hours, and 99.3 overtime hours.

Reductions in duration vary from 18 days to 3 days only when overmanning has the strongest impact on quality and productivity. Identical durations and costs were obtained for all $\varphi \leq 0.40$, except in 2 situations only over 90 cases. $^{3}$ This shows that as long as the impact of overmanning on quality is low, only the effect of overmanning on productivity matters.

In order to better visualize the trade-off between duration and quality, results are rescaled to their maximum value in Fig. 5. The

\footnotetext{
${ }^{3}$ These 2 cases correspond to $\varphi=0.40, \psi=0.80$ and $\rho=2.1$ and 2.2 , with small variations of objective values.
} 

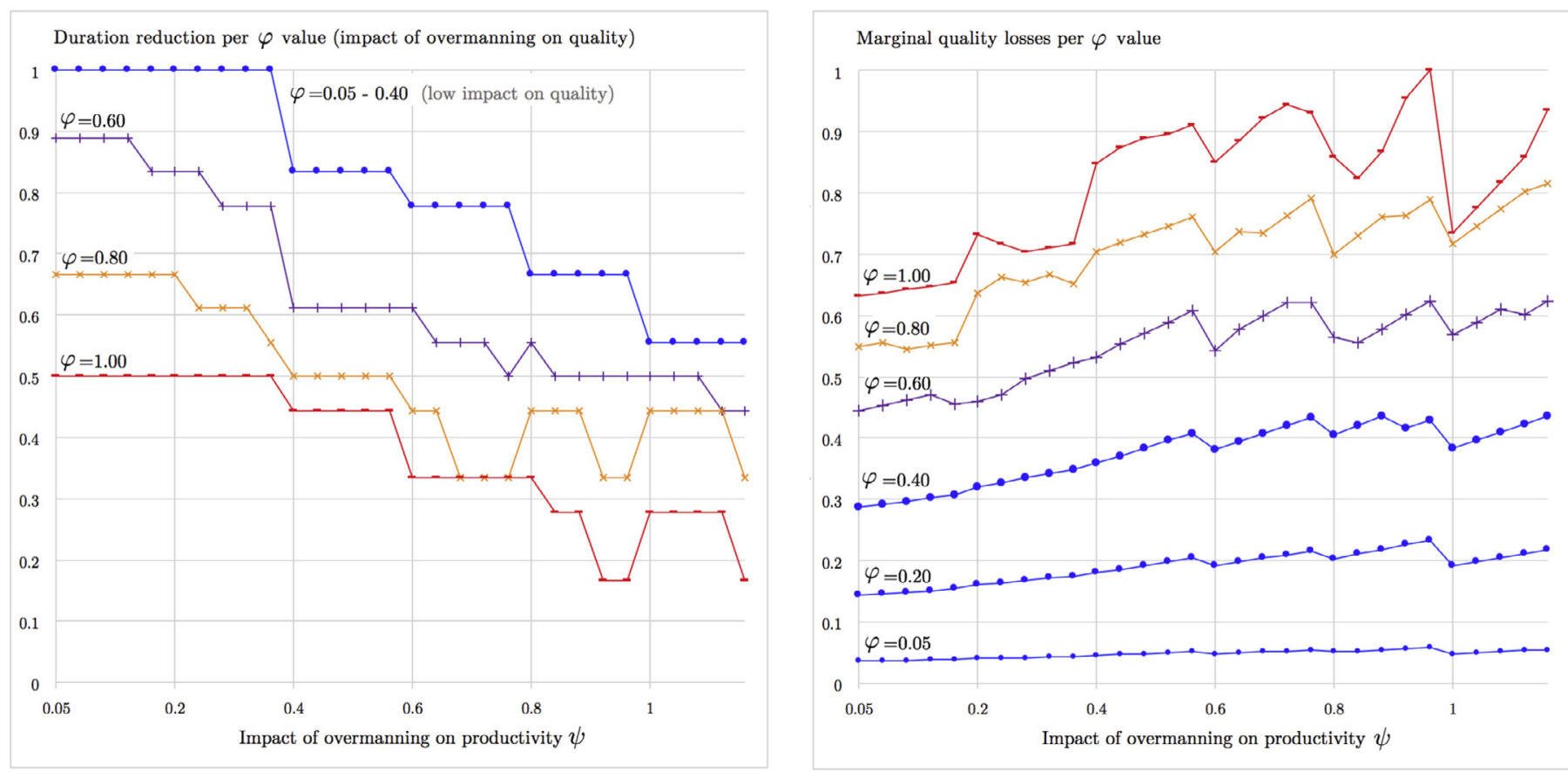

Fig. 5. Duration reduction and marginal quality losses compared with maximum.

left hand side of Fig. 5 plots duration reductions compared with maximum, for each parameters value (for one $\psi$ value, we have five points corresponding to the five values of $\rho$ ). When $\varphi$ is moderate to high $(\varphi \in\{0.60,0.80\})$ as well as in situations where both $\varphi$ and $\psi$ have extreme values $(\varphi=1.00$ and $\psi \geq 0.80)$, the project duration exhibits a sensitivity to $\rho$, due to a quite complex tradeoff between the use of the different kinds of manpower to meet quality constraints.

On the right hand side of Fig. 5, we report the marginal quality loss per day of duration reduction and rescaled to the maximum. Highest marginal quality losses are associated with the lowest duration reductions (and conversely) due to high values of $\varphi$ and $\psi$ that limit acceleration which is made at the expense of important quality losses. Put another way, satisfying individual quality constraints with strong impacts of additional manpower limits duration reductions. On a global basis, as the impact $\varphi$ of overmanning on quality increases quality losses also experience an increase. Parameter $\rho$ also influences the results, outlining the need of a rather accurate estimate of this parameter.

The marginal cost per day of duration reduction is related to the use of additional manpower and to the savings in permanent work. We thus represent in Fig. 6 the evolution of the marginal cost (compared with maximum), and the marginal rates (per day of duration reduction and rescaled to their maximum) of savings in permanent work, utilization of temporary work, and overtime. The dotted blue line on the horizontal axis indicates the value of $\psi$ for which temporary hours are greater than overtime hours.

For a weak impact of overmanning on quality $(\varphi=0.05-0.40$, top left hand side of Fig. 6), more temporary hours than overtime hours are used when overmanning has little influence on productivity $(\psi<0.40)$. As $\psi$ increases above 0.40 , hiring of temporary workers follows the same pattern as savings in permanent workers: when savings decrease, more permanent workers are available which entails a decrease in temporary work and conversely. Overtime continuously increases with $\psi$ but the marginal rate of temporary hours reaches its maximum value for $\psi=1$ because temporary work is needed to offset the higher negative impact of overtime hours on quality. As overtime is more expensive than temporary work, the marginal cost is closely related to the use of overtime.

When the impact of overmanning on quality is medium $(\varphi=$ 0.60 , top right hand side of Fig. 6), more overtime hours than temporary hours are used whatever the impact of overmanning on productivity. Again, hiring of temporary work increases as less permanent work is available. From $\psi \geq 0.40$ overtime usage closely follows permanent work savings. If overmanning strongly affects quality ( $\varphi=0.80$, bottom left hand side of Fig. 6 ), results become very sensitive to $\rho$ values especially when the impact of overmanning on productivity is moderate $(\psi \leq 0.40)$. With additional regular time workers who work relatively fast but with a pretty low quality outcome, the model provides unsteady solutions with large variations of temporary work and overtime usage in an opposite fashion: an important rate of overtime usage is accompanied with a small rate of temporary utilization and conversely. Temporary work reaches its minimum value when $\psi=0.60$ and then increases continuously with $\psi$. Meanwhile, overtime follows the same pattern as permanent work savings, reaching their maximum for identical parameters values.

When the impact of overmanning on quality is maximum $(\varphi=$ 1.00 , bottom right hand side of Fig. 6 ), as long as $\psi \leq 0.60$, more temporary workers than overtime are used, reaching a high utilization rate. For $\psi=0.40-0.60$ results are quite sensitive to $\rho$ values with a volatile overtime utilization, following permanent work savings. For $\psi$ high $(\psi \geq 0.80)$ acceleration ( 3 or 5 days of reduction) is made with overtime only at the smallest marginal cost over all situations. Thus, it is still worth accelerating the project when overmanning strongly affects both the quality and the productivity, but with overtime only.

Our results show the complexity of the trade-off between temporary and overtime hours when quality constraints must be met. Results depend to a large extent on parameters values so the model provides various solutions with quite different usages of additional manpower. With such intertwined effects, one can hardly predict what would be best in terms of labour utilization. This values the contribution of our model for planning properly the different kinds of manpower and provides an incentive to managers 

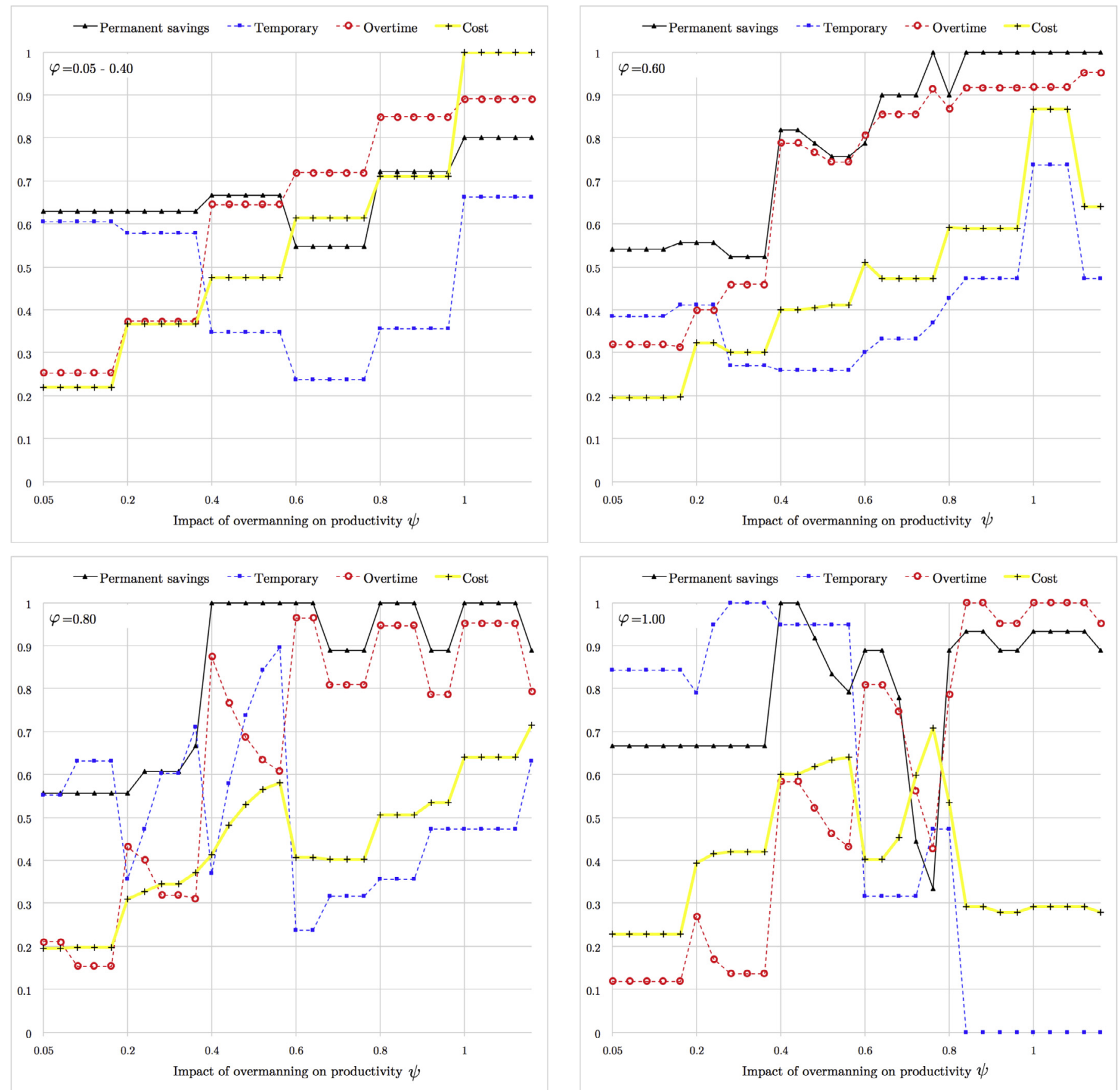

Fig. 6. Marginal costs and marginal rates of permanent workers savings, temporary work and overtime compared with their maximum.

for an accurate estimate of the impact of additional workforce on productivity and on quality.

\subsection{Second phase: efficiency of the MILP formulation}

From our case study, it appears that CPU times heavily depend on parameters values as shown in Fig. 7. The bottom part of this figure provides for each of the 180 instances the total CPU time, and the CPU time to solve each optimization subproblem. In $83 \%$ of the instances (149 out of 180 ), the CPU time to minimize the duration represents the largest proportion of the total time, with $74 \%$ of this total time on average. Next, about $10 \%$ of the instances consume $57 \%$ of the total time to get the optimal cost value at a $0.01 \%$ tolerance level. More rarely ( $7 \%$ of the instances), the objective of minimizing quality losses at a tolerance of $0.1 \%$ takes the longest time to be solved (59\% of the total time on average). The upper part of Fig. 7 plots the average total time over $\rho$-values, making clear the high variability of execution times with respect to parameters $(\psi, \varphi)$. This average reaches a minimum of 375.15 seconds for $(\psi, \varphi)=(0.2,0.05)$ and a maximum of 2971.73 seconds for $(\psi, \varphi)=(0.8,0.2)$. We thus selected these two pairs of parameters values in combination with the 5 values of $\rho$ to define 5 easy to solve instances and 5 hard ones for each of the 6 additional projects. 


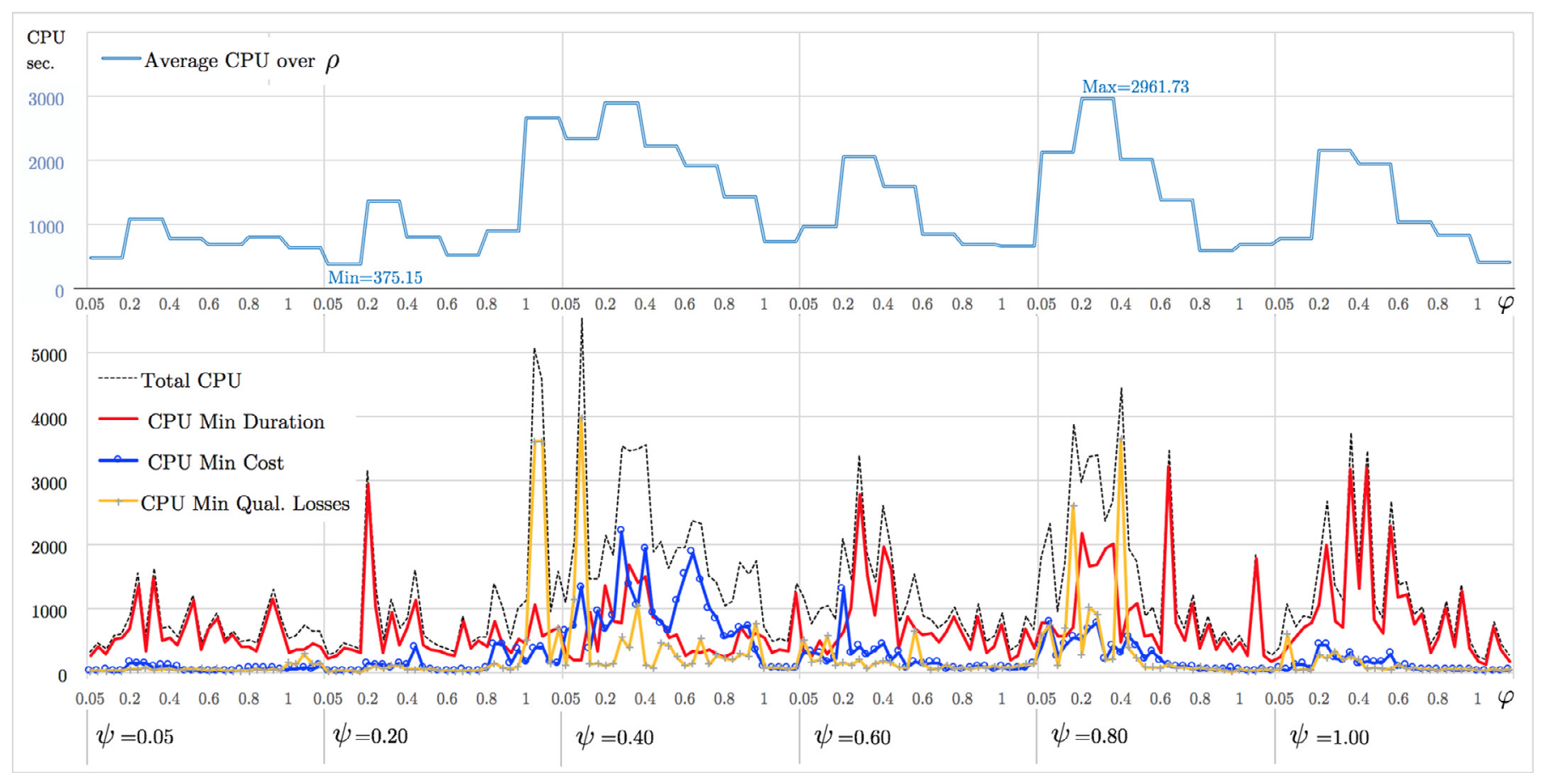

Fig. 7. CPU times of the 180 instances based on the case study.

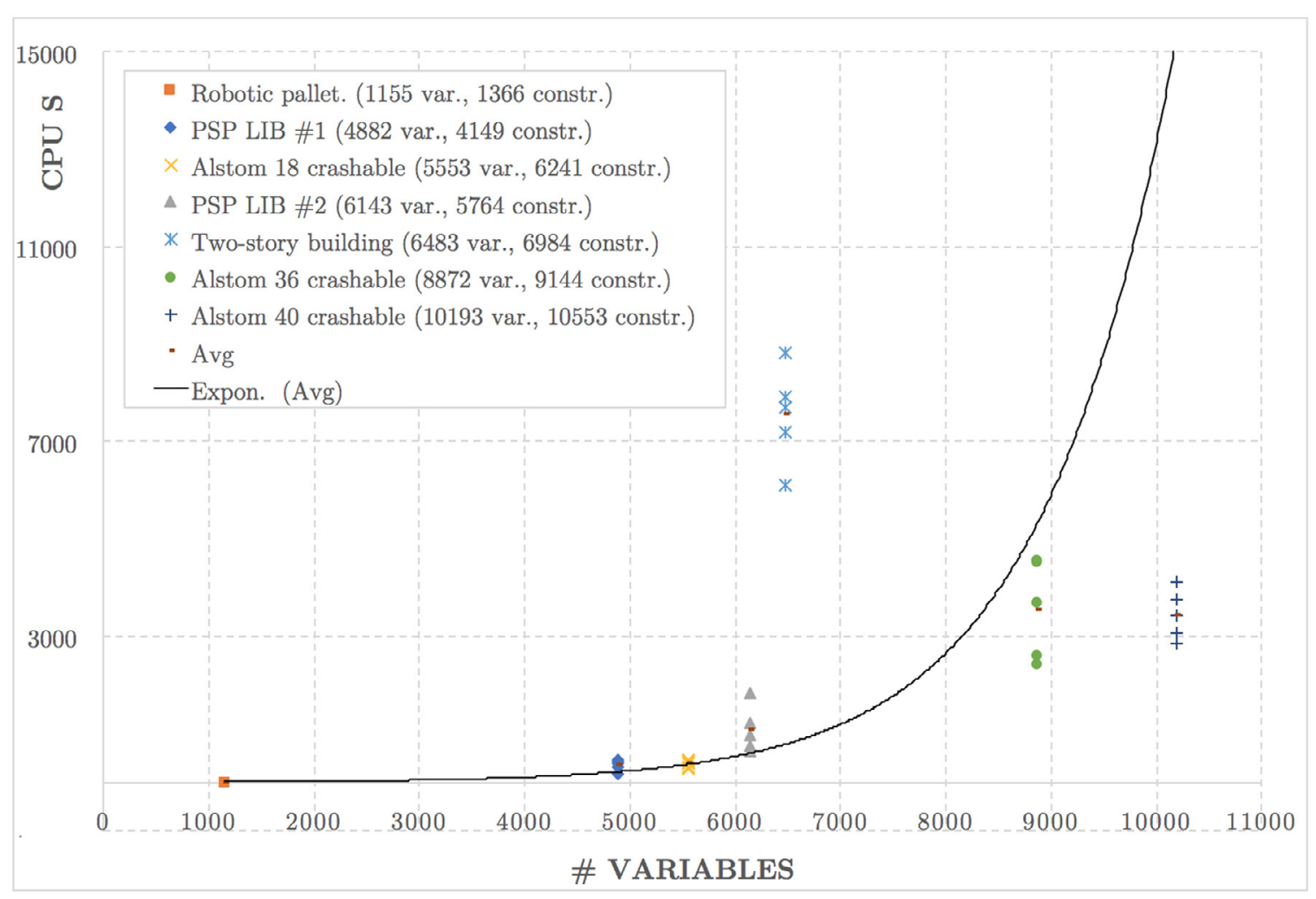

Fig. 8. CPU times of easy instances.

A time limit of $3600 \mathrm{~s}$ per objective to minimize was set for the 30 easy instances. CPLEX reached the optimal solutions in 28 cases out of 30 (at a tolerance level of $0.1 \%$ for the objective of minimizing quality losses). The average gaps to best bound equal $0.59 \%, 0.06 \%$ and $0.19 \%$ for each of the 3 subobjectives respectively, with an average CPU time of 2660.83 seconds per instance. Fig. 8 plots the CPU time to solve the 30 easy instances with respect to the number of variables associated with each project. CPU times obviously increase with the problem size in a non-linear fashion, as shown by the power trend on the average solution times per project.

For hard instances, the time limit per subproblem was increased to 7200 seconds. Beyond a certain number of variables, it was necessary to provide CPLEX with a starting solution. For the 
Table 4

Gap to best bound and execution times - all instances.

\begin{tabular}{|c|c|c|c|c|c|c|c|}
\hline Project description & $\begin{array}{l}\text { Robot type } \\
\text { palletizing } \\
\text { system }\end{array}$ & Random, PSPLIB & $\begin{array}{l}\text { Alstom } 18 \\
\text { cashable } \\
\text { activities }\end{array}$ & Random, PSPLIB & $\begin{array}{l}\text { Two-story } \\
\text { building }\end{array}$ & $\begin{array}{l}\text { Alstom } 36 \\
\text { cashable } \\
\text { activities }\end{array}$ & $\begin{array}{l}\text { Alstom } 40 \\
\text { cashable } \\
\text { activities }\end{array}$ \\
\hline \multicolumn{8}{|l|}{ EASY instances } \\
\hline Duration Gap (\%) & 0 & 0 & 0 & 0 & 0 & 3.579 & 0 \\
\hline Cost Gap (\%) & 0 & 0.008 & 0.009 & 0.009 & 0.343 & 0.005 & 0.006 \\
\hline Quality Gap(\%) & 0 & 0.006 & 0.014 & 0.07 & 0.953 & 0.048 & 0.079 \\
\hline $\mathrm{CPU}(\mathrm{s})$ & 2.729 & 349.967 & 375.153 & 1082.593 & 7551.496 & 3541.274 & 3436.961 \\
\hline \multicolumn{8}{|l|}{ HARD instances } \\
\hline Duration Gap (\%) & 0 & 0 & 0 & 1.951 & 6.136 & 17.066 & 16.309 \\
\hline Cost Gap (\%) & 0 & 0.107 & 0.008 & 0.31 & 0.793 & 0.677 & 0.711 \\
\hline Quality Gap(\%) & 0 & 0.173 & 0.092 & 6.576 & 0.221 & 28.858 & 38.243 \\
\hline $\mathrm{CPU}(\mathrm{s})$ & 2.824 & 12257.241 & 2961.73 & 20788.92 & 21660.524 & 18489.792 & 20266.814 \\
\hline \multicolumn{8}{|l|}{ OVERALL } \\
\hline \#instances & 10 & 10 & 180 & 10 & 10 & 10 & 10 \\
\hline Duration Gap (\%) & 0 & 0 & 0 & 0.976 & 3.068 & 10.323 & 8.154 \\
\hline Cost Gap (\%) & 0 & 0.057 & 0.007 & 0.159 & 0.568 & 0.341 & 0.358 \\
\hline Quality Gap(\%) & 0 & 0.089 & 0.063 & 3.323 & 0.587 & 14.453 & 19.161 \\
\hline $\mathrm{CPU}(\mathrm{s})$ & 2.777 & 6303.604 & 1534.138 & 10935.756 & 14606.01 & 11015.533 & 11851.888 \\
\hline
\end{tabular}

first subproblem of minimizing the makespan, we used the normal mode as an initial solution (no temporary work and no overtime). For the second and third subproblem (cost and quality losses minimization), the starting solution was simply the solution CPLEX found to the previous subproblem. Table 4 provides the average gap to best bound and execution time over the hard instances, the easy ones, and over all instances. With less than 9000 variables and constraints (first five projects), our MILP formulation leads to quite good solutions, with average gaps to best bound less than $3 \%$ for the duration, and $0.6 \%$ for the cost and quality losses. For the last two projects (Alstom, 36 and 40 crashable activities), the average gap to best bound remains quite low for the cost but increases to about $10 \%$ for the duration and to $15-20 \%$ approximately for the quality losses. Beyond 10000 variables, our MILP could hardly be applied as we unsuccessfully tried to solve an instance with 45 activities (extension of our case study). This leaves room for developing a heuristic solution method when larger projects are considered.

\section{Conclusion}

We have developed a MILP formulation to the problem of planning single-skilled manpower with homogeneous and static efficiency to execute a project so as to lexicographically minimize the makespan, the total cost and quality losses while meeting constraints on individual qualities, resource availability as well as nonpreemption and precedence constraints. The rationale for using only additional manpower to expedite the project relies on the fact that workstations (tools and equipment) remain unchanged in the mid-run. Our contribution is three-fold. This paper is the first to formulate a direct relationship between quality and labor utilization. Second, we consider productivity losses due to overmanning. Third, our model needs an estimate for only three parameters for each activity to obtain the cost, the quality and the duration associated with an almost infinite number of execution modes, which makes its implementation in practice easier than multimode modeling approaches requiring parameters estimation in each activity execution mode.

The model provides the optimal allocation of permanent and temporary workers as well as overtime hours to each processing period of every activities where flexible resource profiles are assumed, which is consistent with project management practices in manufacturing environments. Based on a real project of a very high speed train motor coach at Alstom, 180 instances have been generated on which the model has been successfully implemented with optimal manpower allocation showing a complex trade-off between permanent work savings, temporary workers and overtime hours. Besides, we have shown the applicability of our model to other types of projects.

Future research includes an empirical study to establish the relationship between additional manpower (overtime and overmanning) and productivity on the one hand and between manpower and quality on the other hand, for several work types and manufacturing environments. The efficiency of other MIP formulations could be explored as in Naber and Kolisch (2014) and heuristic solution methods could be developed.

\section{Acknowledgments}

The following managers at Alstom are kindly acknowledged for providing the data of the case study and their expertise to appraise our assumptions: N. Bertrand (Production and Supply Chain Manager); S. El Bouayadi (Production Quality Manager); S. Tiouiri (Suppliers' Quality Manager); N. Sanyoura (Electrical Test Engineer) and O. Arikaya (Production Sourcing Manager).

We express our gratitude to three anonymous reviewers for their constructive comments. 
Appendix A. Notations and definitions

Table 5

Notations and definitions.

\begin{tabular}{|c|c|}
\hline \multicolumn{2}{|l|}{ Indices } \\
\hline$i, j$ & Activity \\
\hline$t, \tau$ & Time period (day) \\
\hline$k$ & Skill \\
\hline \multicolumn{2}{|l|}{ Parameters } \\
\hline$V=\{0, \ldots, n+1\}$ & Set of activities ( $n$ is the number of actual activities) \\
\hline$\Gamma^{-1}(j), \Gamma(j)$ & Set of activities immediately preceding $j$ and succeeding $j$ respectively \\
\hline$K$ & Number of different skills required for the project \\
\hline$V_{k}$ & Subset of activities requiring skill $k$ \\
\hline$P_{j}^{\text {nor }}, P_{j}^{\max }$ & Normal and max. number of workers with skill $k$ on $j$ in each period \\
\hline$d_{j}^{\text {nor }}$ & Normal processing time of activity $j$ \\
\hline$\gamma_{j}$ & Impact of overmanning on productivity losses for activity $j$ \\
\hline$q_{j}^{\text {nor }}, q_{j}^{\min }$ & Normal quality and quality threshold for activity $j$ \\
\hline$\lambda_{j}^{R}$ & Quality loss of $j$ induced by one worker in overmanning for one period \\
\hline$\lambda_{j}^{0}$ & Quality loss of $j$ associated with one overtime hour \\
\hline$H_{R}$ & Number of regular work hours per worker and period \\
\hline$H_{O}, H_{O W}$ & Max. number of overtime hours per worker, per day and per week resp. \\
\hline$c_{P}, c_{U}, c_{O}$ & Cost of one hour of permanent work, temporary work and overtime resp. \\
\hline \multicolumn{2}{|l|}{ Computed parameters } \\
\hline $\mathcal{P}_{k}$ & Total number of workers with skill $k$ dedicated to the project \\
\hline$\underline{\mathrm{d}}_{j}$ & Lower bound on the processing time of activity $j$ \\
\hline $\overrightarrow{E S}, L F_{j}$ & Earliest start time and latest finish time of activity $j$ \\
\hline$C_{\max }^{\text {nor }}, \underline{C}_{\max }$ & Upper and lower bounds on the makespan \\
\hline$W_{j}^{R}(l)$ & Work content of $l$ workers on activity $j, l=0 . . P_{j}^{\max }$ \\
\hline$L_{j}^{R}(l)$ & Quality loss associated with $l$ workers on activity $j, l=0 . . P_{j}^{\max }$ \\
\hline \multicolumn{2}{|l|}{ Variables } \\
\hline$C_{\max }$ & Project completion time \\
\hline$p_{j t}$ & Number of permanent workers allocated to $j$ in period $t$ (integer) \\
\hline$u_{j t}$ & Number of temporary workers allocated to $j$ in period $t$ (integer) \\
\hline$h_{j t}$ & Number of overtime hours assigned to $j$ in period $t$ (continuous) \\
\hline$z_{j t}$ & Binary equal to 1 if $j$ is processed in period $t$ and 0 otherwise \\
\hline$y_{j t l}$ & Binary equal to 1 if $p_{j t}+u_{j t}=l$ and 0 otherwise \\
\hline \multicolumn{2}{|l|}{ Definitions } \\
\hline $\mathcal{T}_{j}=\left\{E S_{j}, \ldots, L F_{j}\right\}$ & Set of feasible processing periods for activity $j$ \\
\hline$W_{j t}^{R} \equiv W_{j}^{R}\left(p_{j t}+u_{j t}\right)$ & Number of productive workers during r...egular hours on activity $j$ in period $t$ \\
\hline$W_{j t}^{O} \equiv W_{j}^{O}\left(h_{j t}\right)$ & Number of productive workers during overtime hours on activity $j$ in period $t$ \\
\hline$L_{j t}^{R} \equiv L_{j}^{R}\left(p_{j t}+u_{j t} ; \lambda_{j}^{R}\right)$ & Quality loss of $j$ in period $t$ due to overmanning \\
\hline$L_{j t}^{O} \equiv L_{j}^{O}\left(h_{j t} ; \lambda_{j}^{O}\right)$ & Quality loss of $j$ in period $t$ due to overtime \\
\hline$q_{j}$ & Quality of activity $j$ \\
\hline$L$ & Project quality loss \\
\hline$B$ & Project total cost \\
\hline \multicolumn{2}{|c|}{ Parameters in the simulations } \\
\hline$\psi$ with $\gamma_{j} \equiv \gamma_{j}(\psi)$ & General impact of overmanning on productivity in the company \\
\hline$\varphi$ with $\lambda_{j}^{R} \equiv \lambda_{j}^{R}(\varphi)$ & General impact of overmanning on quality in the company \\
\hline$\rho$ with $\lambda_{j}^{O} \equiv \lambda_{j}^{O}(\rho)$ & General impact of overtime on quality in the company \\
\hline
\end{tabular}

\section{Appendix B. Data of the project at Alstom}

Table 6

Quality control inspection checklist for activity $j=6$ : Motor coach painting.

\begin{tabular}{lllll}
\hline Item \# & Check point & Defect statement & Impact & Acceptability \\
\hline 1 & Painting area & Overlap/Missing & Non compliance with customer specifications & No \\
2 & Color hue & Major defect & Non compliance with customer specifications & No \\
3 & Color hue & Small localized defect & Esthetics & Yes \\
4 & Resin finishing & Non uniformity & Non compliance with customer specifications & No \\
5 & Resin area & Missing & Non compliance with customer specifications & No \\
6 & Paint finishing & Missing & Non compliance with customer specifications & No \\
7 & Paint finishing & Invisible peel-off & Esthetics & Yes \\
8 & Paint finishing & Light scratches & Non compliance with customer specifications & No \\
9 & Paint composition & Wrong percentage of components & &
\end{tabular}


Table 7

Parameters of the project activities.

\begin{tabular}{|c|c|c|c|c|c|c|c|}
\hline$j$ & Description & $\Gamma^{-1}(j)$ & $k$ & $d_{j}^{\text {nor }}$ & $P_{j}^{\text {nor }}$ & $P_{j}^{\max }$ & $q_{j}^{\min }$ \\
\hline 0 & Start & $\emptyset$ & - & 0 & - & - & - \\
\hline 1 & Girder welding & 0 & 1 & 7 & 4 & 5 & 0.80 \\
\hline 2 & Lower chassis welding & 1 & 1 & 7 & 4 & 5 & 0.80 \\
\hline 3 & Left lateral side mounting & 2 & 1 & 7 & 3 & 4 & 0.80 \\
\hline 4 & Right lateral side mounting & 3 & 1 & 7 & 3 & 4 & 0.80 \\
\hline 5 & Cabin assembly & 4 & 1 & 8 & 3 & 4 & 0.80 \\
\hline 6 & Motor coach painting & 5 & 2 & 10 & 3 & 4 & 0.67 \\
\hline 7 & Piping mechanical parts & 6 & 3 & 4 & 4 & 5 & 0.80 \\
\hline 8 & High voltage pre-cabling & 0 & 4 & 9 & 1 & 2 & 0.80 \\
\hline 9 & High voltage cabling & 7,8 & 3 & 1 & 4 & 4 & 0.80 \\
\hline 10 & Floor and ventilation ducts & 9 & 3 & 1 & 4 & 4 & 0.80 \\
\hline 11 & Low voltage precabling & 0 & 4 & 24 & 1 & 2 & 0.80 \\
\hline 12 & Low voltage cabling & 10,11 & 3 & 2 & 4 & 5 & 0.80 \\
\hline 13 & Front part cabling & 12 & 3 & 1 & 2 & 2 & 0.80 \\
\hline 14 & Fairing and trapdoors equipment & 12 & 3 & 1 & 3 & 3 & 0.67 \\
\hline 15 & Fairing and windscreen & 13,14 & 3 & 1 & 5 & 5 & 0.80 \\
\hline 16 & Driver desk and cabin covers & 15 & 3 & 1 & 5 & 5 & 0.80 \\
\hline 17 & Cabinet partition and tools & 16 & 3 & 1 & 5 & 5 & 0.80 \\
\hline 18 & Indoor section & 17 & 3 & 2 & 5 & 6 & 0.80 \\
\hline 19 & Engine and underframe equipment & 18 & 3 & 2 & 5 & 6 & 0.80 \\
\hline 20 & Cowls connection & 19 & 5 & 6 & 1 & 2 & 0.80 \\
\hline 21 & Cowls equipment & 19 & 5 & 6 & 1 & 2 & 0.67 \\
\hline 22 & Cowls mounting & 20,21 & 5 & 2 & 2 & 3 & 0.80 \\
\hline 23 & Cabling/balancing & 22 & 5 & 1 & 2 & 2 & 0.80 \\
\hline 24 & Rooftop equipment & 23 & 5 & 1 & 2 & 2 & 0.67 \\
\hline 25 & Air brake test & 24 & 3 & 1 & 2 & 2 & 1.00 \\
\hline 26 & Chocks laying & 25 & 3 & 1 & 2 & 2 & 0.67 \\
\hline 27 & Bogie mounting preparation & 26 & 3 & 2 & 2 & 3 & 0.67 \\
\hline 28 & Bogie mounting and connection & 27 & 3 & 2 & 2 & 3 & 0.80 \\
\hline 29 & Louvers & 28 & 3 & 3 & 2 & 3 & 0.80 \\
\hline 30 & Low voltage test & 29 & 5 & 11 & 2 & 2 & 1.00 \\
\hline 31 & High voltage test & 30 & 5 & 2 & 2 & 2 & 1.00 \\
\hline 32 & Pneumatic test & 31 & 6 & 4 & 2 & 2 & 1.00 \\
\hline 33 & Safety equipment test & 32 & 7 & 5 & 2 & 2 & 1.00 \\
\hline 34 & Siemens supplies test & 33 & 7 & 2 & 2 & 2 & 1.00 \\
\hline 35 & Dynamic test & 34 & 7 & 1 & 2 & 2 & 1.00 \\
\hline 36 & Mechanical test & 35 & 7 & 1 & 2 & 2 & 1.00 \\
\hline 37 & End & 36 & - & 0 & - & - & - \\
\hline
\end{tabular}

Legend. Skill $k=1$ : welding; $k=2$ : painting; $k=3$ : assembly; $k=4$ : wiring; $k=5$ : electricity; $k=6$ : pneumatic; $k=7$ : electronic automation.

\section{Appendix C. Estimation of parameters}

It can be possible to produce an estimate of coefficient $\gamma_{j}$ by allocating a constant number of workers $p_{j}+u_{j}=P_{j}^{\text {nor }}+1$ over processing periods and recording the resultant durations $d_{j}^{\text {obs }}$. Using Eq. (26) with no overtime hours, we would have $d_{j}^{\text {obs }}=\left(P_{j}^{\text {nor }}\right.$. $\left.d_{j}^{\text {nor }}\right) / W_{j}^{R}\left(P_{j}^{\text {nor }}+1\right)$. Replacing $W_{j}^{R}\left(P_{j}^{\text {nor }}+1\right)$ by its expression in Eq. (4), we get $d_{j}^{\text {obs }}=P_{j}^{\text {nor }} \cdot d_{j}^{\text {nor }} /\left[\left(1+\gamma_{j}\right) \cdot\left(P_{j}^{\text {nor }}+1\right)-\frac{\gamma_{j}}{P_{j}^{\text {or }}} \cdot\left(P_{j}^{\text {nor }}+\right.\right.$ $1)^{2}$ ] that we can solve in $\gamma_{j}$ to obtain an estimate $\hat{\gamma}_{j}=P_{j}^{\text {nor }}-$ $\left(\left(P_{j}^{\text {nor }}\right)^{2} /\left(P_{j}^{\text {nor }}+1\right)\right) \cdot\left(d_{j}^{\text {nor }} / d_{j}^{\text {obs }}\right)$. Recording the observed quality $q_{j}^{\text {obs }}$ in the same overmanning situation and using Eq. (10), we obtain $q_{j}^{\text {obs }}=q_{j}^{\text {nor }}-\lambda_{j}^{R} \cdot d_{j}^{\text {obs }}$ which provides an estimate $\lambda_{j}^{R}=\left(q_{j}^{\text {nor }}-\right.$ $\left.q_{j}^{\text {obs }}\right) / d_{j}^{\text {obs }}$.

Following a similar reasoning, it could be possible to record quality $q_{j}^{\text {obs }}$ associated with a constant number of overtime hours $h_{j}$ over processing periods and no overmanning. With Eq. (26), the processing time $d_{j}$ would equal $d_{j}=P_{j}^{\text {nor }} \cdot d_{j}^{\text {nor }} /\left(P_{j}^{\text {nor }}+h_{j} / H_{R}\right)$. Using Eq. (10), we get $q_{j}^{\text {obs }}=q_{j}^{\text {nor }}-\lambda_{j}^{O} \cdot d_{j} \cdot h_{j}$ which leads to $\hat{\lambda_{j}^{O}}=$ $\left(q_{j}^{\text {nor }}-q_{j}^{\text {obs }}\right) /\left(d_{j} \cdot h_{j}\right)$. Obviously, repeating the experiment a large number of times would allow for sharper estimates.

\section{Appendix D. Simulation results}

Let us recall that parameter $\psi$ reflects the impact of overmanning on productivity losses, parameter $\varphi$ denotes the impact of overmanning on quality and parameter $\rho$ measures the impact on quality of overtime relatively to overmanning ( $\rho=1.8$ for instance means that the impact of one overtime hour on quality is 1.8 times higher than the impact of one hour of overmanning on the quality). Blue shaded cells show that we have the same duration reductions and cost increases for $\varphi \in\{0.05,0.20,0.40\}$ and for each $\psi$ and $\rho$ values (except in 2 situations over 90 with $\psi=0.80 ; \varphi=0.40$ and $\rho \in\{2.1,2.2\}$ ). 
Table 8

Simulation results for instances \#1 to \#90.

\begin{tabular}{|c|c|c|c|c|c|c|c|c|c|}
\hline \multicolumn{4}{|c|}{ Instance } & \multicolumn{3}{|c|}{ Variation in $\%$} & \multicolumn{3}{|c|}{ Total number of hours } \\
\hline$\#$ & $\psi$ & $\varphi$ & $\rho$ & Duration & Cost & Quality & Perm. saving & Temporary & Overtime \\
\hline 1 & 0.05 & 0.05 & 1.8 & -16.82 & 2.74 & -0.43 & 265.2 & 179.4 & 99.3 \\
\hline 2 & & & 1.9 & -16.82 & 2.74 & -0.43 & 265.2 & 179.4 & 99.3 \\
\hline 3 & & & 2.0 & -16.82 & 2.74 & -0.44 & 265.2 & 179.4 & 99.3 \\
\hline 4 & & & 2.1 & -16.82 & 2.74 & -0.45 & 265.2 & 179.4 & 99.3 \\
\hline 5 & & & 2.2 & -16.82 & 2.74 & -0.46 & 265.2 & 179.4 & 99.3 \\
\hline 6 & & 0.20 & 1.8 & -16.82 & 2.74 & -1.71 & 265.2 & 179.4 & 99.3 \\
\hline 7 & & & 1.9 & -16.82 & 2.74 & -1.74 & 265.2 & 179.4 & 99.3 \\
\hline 8 & & & 2.0 & -16.82 & 2.74 & -1.77 & 265.2 & 179.4 & 99.3 \\
\hline 9 & & & 2.1 & -16.82 & 2.74 & -1.79 & 265.2 & 179.4 & 99.3 \\
\hline 10 & & & 2.2 & -16.82 & 2.74 & -1.82 & 265.2 & 179.4 & 99.3 \\
\hline 11 & & 0.40 & 1.8 & -16.82 & 2.74 & $\overline{-3.42}$ & 265.2 & 179.4 & 99.3 \\
\hline 12 & & & 1.9 & -16.82 & 2.74 & -3.47 & 265.2 & 179.4 & 99.3 \\
\hline 13 & & & 2.0 & -16.82 & 2.74 & -3.53 & 265.2 & 179.4 & 99.3 \\
\hline 14 & & & 2.1 & -16.82 & 2.74 & -3.59 & 265.2 & 179.4 & 99.3 \\
\hline 15 & & & 2.2 & -16.82 & 2.74 & -3.65 & 265.2 & 179.4 & 99.3 \\
\hline 16 & & 0.60 & 1.8 & -14.95 & 2.16 & -4.70 & 202.8 & 101.4 & 111.7 \\
\hline 17 & & & 1.9 & -14.95 & 2.16 & -4.80 & 202.8 & 101.4 & 111.7 \\
\hline 18 & & & 2.0 & -14.95 & 2.16 & -4.89 & 202.8 & 101.4 & 111.7 \\
\hline 19 & & & 2.1 & -14.95 & 2.16 & -4.99 & 202.8 & 101.4 & 111.7 \\
\hline 20 & & & 2.2 & -14.02 & 2.04 & -4.52 & 195.0 & 101.4 & 102.9 \\
\hline 21 & & 0.80 & 1.8 & -11.21 & 1.63 & -4.35 & 156.0 & 109.2 & 55.2 \\
\hline 22 & & & 1.9 & -11.21 & 1.63 & -4.41 & 156.0 & 109.2 & 55.2 \\
\hline 23 & & & 2.0 & -11.21 & 1.63 & -4.32 & 156.0 & 124.8 & 40.3 \\
\hline 24 & & & 2.1 & -11.21 & 1.63 & -4.37 & 156.0 & 124.8 & 40.3 \\
\hline 25 & & & 2.2 & -11.21 & 1.63 & -4.41 & 156.0 & 124.8 & 40.3 \\
\hline 26 & & 1.00 & 1.8 & -8.41 & 1.42 & -3.76 & 140.4 & 124.8 & 23.3 \\
\hline 27 & & & 1.9 & -8.41 & 1.42 & -3.79 & 140.4 & 124.8 & 23.3 \\
\hline 28 & & & 2.0 & -8.41 & 1.42 & -3.82 & 140.4 & 124.8 & 23.3 \\
\hline 29 & & & 2.1 & -8.41 & 1.42 & -3.85 & 140.4 & 124.8 & 23.3 \\
\hline 30 & & & 2.2 & -8.41 & 1.42 & -3.89 & 140.4 & 124.8 & 23.3 \\
\hline 31 & 0.20 & 0.05 & 1.8 & -16.82 & 4.58 & -0.48 & 265.2 & 171.6 & 146.5 \\
\hline 32 & & & 1.9 & -16.82 & 4.58 & -0.49 & 265.2 & 171.6 & 146.5 \\
\hline 33 & & & 2.0 & -16.82 & 4.58 & -0.50 & 265.2 & 171.6 & 146.5 \\
\hline 34 & & & 2.1 & -16.82 & 4.58 & -0.51 & 265.2 & 171.6 & 146.5 \\
\hline 35 & & & 2.2 & -16.82 & 4.58 & -0.52 & 265.2 & 171.6 & 146.5 \\
\hline 36 & & 0.20 & 1.8 & -16.82 & 4.58 & -1.90 & 265.2 & 171.6 & 146.5 \\
\hline 37 & & & 1.9 & -16.82 & 4.58 & -1.95 & 265.2 & 171.6 & 146.5 \\
\hline 38 & & & 2.0 & -16.82 & 4.58 & -1.99 & 265.2 & 171.6 & 146.5 \\
\hline 39 & & & 2.1 & -16.82 & 4.58 & -2.03 & 265.2 & 171.6 & 146.5 \\
\hline 40 & & & 2.2 & -16.82 & 4.58 & -2.08 & 265.2 & 171.6 & 146.5 \\
\hline 41 & & 0.40 & 1.8 & -16.82 & 4.58 & -3.81 & 265.2 & 171.6 & 146.5 \\
\hline 42 & & & 1.9 & -16.82 & 4.58 & -3.89 & 265.2 & 171.6 & 146.5 \\
\hline 43 & & & 2.0 & -16.82 & 4.58 & -3.98 & 265.2 & 171.6 & 146.5 \\
\hline 44 & & & 2.1 & -16.82 & 4.58 & -4.07 & 265.2 & 171.6 & 146.5 \\
\hline 45 & & & 2.2 & -16.82 & 4.58 & -4.15 & 265.2 & 171.6 & 146.5 \\
\hline
\end{tabular}

\begin{tabular}{|c|c|c|c|c|c|c|c|c|c|}
\hline \multicolumn{4}{|c|}{ Instance (continued) } & \multicolumn{3}{|c|}{ Variation in $\%$} & \multicolumn{3}{|c|}{ Total number of hours } \\
\hline$\#$ & $\psi$ & $\varphi$ & $\rho$ & Duration & Cost & Quality & Perm. saving & Temporary & Overtime \\
\hline 46 & 0.20 & 0.60 & 1.8 & -14.02 & 3.34 & -4.56 & 195.0 & 101.4 & 130.9 \\
\hline 47 & & & 1.9 & -14.02 & 3.34 & -4.67 & 195.0 & 101.4 & 130.9 \\
\hline 48 & & & 2.0 & -13.08 & 2.90 & -4.60 & 171.6 & 62.4 & 140.2 \\
\hline 49 & & & 2.1 & -13.08 & 2.90 & -4.72 & 171.6 & 62.4 & 140.2 \\
\hline 50 & & & 2.2 & -13.08 & 2.90 & -4.83 & 171.6 & 62.4 & 140.2 \\
\hline 51 & & 0.80 & 1.8 & -11.21 & 2.57 & -5.05 & 156.0 & 70.2 & 113.1 \\
\hline 52 & & & 1.9 & -10.28 & 2.49 & -4.81 & 156.0 & 85.8 & 96.4 \\
\hline 53 & & & 2.0 & -10.28 & 2.62 & -4.76 & 156.0 & 109.2 & 76.8 \\
\hline 54 & & & 2.1 & -10.28 & 2.62 & -4.85 & 156.0 & 109.2 & 76.8 \\
\hline 55 & & & 2.2 & -9.35 & 2.56 & -4.30 & 156.0 & 117.0 & 67.9 \\
\hline 56 & & 1.00 & 1.8 & -8.41 & 2.45 & -4.36 & 140.4 & 117.0 & 53.0 \\
\hline 57 & & & 1.9 & -8.41 & 2.58 & -4.26 & 140.4 & 140.4 & 33.5 \\
\hline 58 & & & 2.0 & -8.41 & 2.62 & -4.19 & 140.4 & 148.2 & 26.7 \\
\hline 59 & & & 2.1 & -8.41 & 2.62 & -4.23 & 140.4 & 148.2 & 26.7 \\
\hline 60 & & & 2.2 & -8.41 & 2.62 & -4.27 & 140.4 & 148.2 & 26.7 \\
\hline 61 & 0.40 & 0.05 & 1.8 & -14.02 & 4.92 & -0.45 & 234.0 & 85.8 & 211.3 \\
\hline 62 & & & 1.9 & -14.02 & 4.92 & -0.46 & 234.0 & 85.8 & 211.3 \\
\hline 63 & & & 2.0 & -14.02 & 4.92 & -0.48 & 234.0 & 85.8 & 211.3 \\
\hline 64 & & & 2.1 & -14.02 & 4.92 & -0.49 & 234.0 & 85.8 & 211.3 \\
\hline 65 & & & 2.2 & -14.02 & 4.92 & -0.51 & 234.0 & 85.8 & 211.3 \\
\hline 66 & & 0.20 & 1.8 & -14.02 & 4.92 & -1.78 & 234.0 & 85.8 & 211.3 \\
\hline 67 & & & 1.9 & -14.02 & 4.92 & -1.84 & 234.0 & 85.8 & 211.3 \\
\hline 68 & & & 2.0 & -14.02 & 4.92 & -1.90 & 234.0 & 85.8 & 211.3 \\
\hline 69 & & & 2.1 & -14.02 & 4.92 & -1.96 & 234.0 & 85.8 & 211.3 \\
\hline 70 & & & 2.2 & -14.02 & 4.92 & -2.02 & 234.0 & 85.8 & 211.3 \\
\hline 71 & & 0.40 & 1.8 & -14.02 & 4.92 & -3.56 & 234.0 & 85.8 & 211.3 \\
\hline 72 & & & 1.9 & -14.02 & 4.92 & -3.68 & 234.0 & 85.8 & 211.3 \\
\hline 73 & & & 2.0 & -14.02 & 4.92 & -3.80 & 234.0 & 85.8 & 211.3 \\
\hline 74 & & & 2.1 & -14.02 & 4.92 & -3.92 & 234.0 & 85.8 & 211.3 \\
\hline 75 & & & 2.2 & -14.02 & 4.92 & -4.04 & 234.0 & 85.8 & 211.3 \\
\hline 76 & & 0.60 & 1.8 & -10.28 & 3.04 & -3.86 & 210.6 & 46.8 & 189.3 \\
\hline 77 & & & 1.9 & -10.28 & 3.04 & -4.02 & 210.6 & 46.8 & 189.3 \\
\hline 78 & & & 2.0 & -10.28 & 3.08 & -4.15 & 202.8 & 46.8 & 184.0 \\
\hline 79 & & & 2.1 & -10.28 & 3.12 & -4.28 & 195.0 & 46.8 & 178.7 \\
\hline 80 & & & 2.2 & -10.28 & 3.12 & -4.43 & 195.0 & 46.8 & 178.7 \\
\hline 81 & & 0.80 & 1.8 & -8.41 & 2.57 & -4.19 & 210.6 & 54.6 & 171.8 \\
\hline 82 & & & 1.9 & -8.41 & 2.99 & -4.28 & 210.6 & 85.8 & 150.8 \\
\hline 83 & & & 2.0 & -8.41 & 3.30 & -4.36 & 210.6 & 109.2 & 135.1 \\
\hline 84 & & & 2.1 & -8.41 & 3.51 & -4.44 & 210.6 & 124.8 & 124.7 \\
\hline 85 & & & 2.2 & -8.41 & 3.61 & -4.53 & 210.6 & 132.6 & 119.4 \\
\hline 86 & & 1.00 & 1.8 & -7.48 & 3.32 & -4.49 & 187.2 & 124.8 & 101.9 \\
\hline 87 & & & 1.9 & -7.48 & 3.32 & -4.63 & 187.2 & 124.8 & 101.9 \\
\hline 88 & & & 2.0 & -7.48 & 3.41 & -4.70 & 171.6 & 124.8 & 91.3 \\
\hline 89 & & & 2.1 & -7.48 & 3.50 & -4.74 & 156.0 & 124.8 & 80.7 \\
\hline 90 & & & 2.2 & -7.48 & 3.54 & -4.81 & 148.2 & 124.8 & 75.4 \\
\hline
\end{tabular}


Table 9

Simulation results for instances \#91 to \#180

\begin{tabular}{|c|c|c|c|c|c|c|c|c|c|}
\hline \multicolumn{4}{|c|}{ Instance (continued) } & \multicolumn{3}{|c|}{ Variation in $\%$} & \multicolumn{3}{|c|}{ Total number of hours } \\
\hline$\#$ & $\psi$ & $\varphi$ & $\rho$ & Duration & Cost & Quality & Perm. saving & Temporary & Overtime \\
\hline 91 & 0.60 & 0.05 & 1.8 & -13.08 & 5.94 & -0.44 & 179.4 & 54.6 & 219.6 \\
\hline 92 & & & 1.9 & -13.08 & 5.94 & -0.46 & 179.4 & 54.6 & 219.6 \\
\hline 93 & & & 2.0 & -13.08 & 5.94 & -0.47 & 179.4 & 54.6 & 219.6 \\
\hline 94 & & & 2.1 & -13.08 & 5.94 & -0.49 & 179.4 & 54.6 & 219.6 \\
\hline 95 & & & 2.2 & -13.08 & 5.94 & -0.50 & 179.4 & 54.6 & 219.6 \\
\hline 96 & & 0.20 & 1.8 & -13.08 & 5.94 & -1.76 & 179.4 & 54.6 & 219.6 \\
\hline 97 & & & 1.9 & -13.08 & 5.94 & -1.82 & 179.4 & 54.6 & 219.6 \\
\hline 98 & & & 2.0 & -13.08 & 5.94 & -1.88 & 179.4 & 54.6 & 219.6 \\
\hline 99 & & & 2.1 & -13.08 & 5.94 & -1.94 & 179.4 & 54.6 & 219.6 \\
\hline 100 & & & 2.2 & -13.08 & 5.94 & -2.00 & 179.4 & 54.6 & 219.6 \\
\hline 101 & & 0.40 & 1.8 & -13.08 & 5.94 & -3.52 & 179.4 & 54.6 & 219.6 \\
\hline 102 & & & 1.9 & -13.08 & 5.94 & -3.64 & 179.4 & 54.6 & 219.6 \\
\hline 103 & & & 2.0 & -13.08 & 5.94 & -3.76 & 179.4 & 54.6 & 219.6 \\
\hline 104 & & & 2.1 & -13.08 & 5.94 & -3.88 & 179.4 & 54.6 & 219.6 \\
\hline 105 & & & 2.2 & -13.08 & 5.94 & -4.00 & 179.4 & 54.6 & 219.6 \\
\hline 106 & & 0.60 & 1.8 & -10.28 & 3.88 & -3.94 & 202.8 & 54.6 & 193.9 \\
\hline 107 & & & 1.9 & -9.35 & 3.26 & -3.81 & 210.6 & 54.6 & 186.7 \\
\hline 108 & & & 2.0 & -9.35 & 3.26 & -3.96 & 210.6 & 54.6 & 186.7 \\
\hline 109 & & & 2.1 & -9.35 & 3.26 & -4.11 & 210.6 & 54.6 & 186.7 \\
\hline 110 & & & 2.2 & -8.41 & 2.94 & -3.69 & 210.6 & 54.6 & 179.7 \\
\hline 111 & & 0.80 & 1.8 & -7.48 & 2.25 & -3.72 & 187.2 & 31.2 & 168.5 \\
\hline 112 & & & 1.9 & -7.48 & 2.25 & -3.89 & 187.2 & 31.2 & 168.5 \\
\hline 113 & & & 2.0 & -5.61 & 1.67 & -2.92 & 124.8 & 31.2 & 106.1 \\
\hline 114 & & & 2.1 & -5.61 & 1.67 & -3.03 & 124.8 & 31.2 & 106.1 \\
\hline 115 & & & 2.2 & -5.61 & 1.67 & -3.14 & 124.8 & 31.2 & 106.1 \\
\hline 116 & & 1.00 & 1.8 & -5.61 & 1.67 & -3.37 & 124.8 & 31.2 & 106.1 \\
\hline 117 & & & 1.9 & -5.61 & 1.67 & -3.51 & 124.8 & 31.2 & 106.1 \\
\hline 118 & & & 2.0 & -5.61 & 1.87 & -3.66 & 109.2 & 31.2 & 98.0 \\
\hline 119 & & & 2.1 & -5.61 & 2.48 & -3.74 & 62.4 & 31.2 & 73.6 \\
\hline 120 & & & 2.2 & -5.61 & 2.94 & -3.69 & 46.8 & 46.8 & 56.2 \\
\hline 121 & 0.80 & 0.05 & 1.8 & -11.21 & 5.89 & -0.40 & 202.8 & 70.2 & 222.5 \\
\hline 122 & & & 1.9 & -11.21 & 5.89 & -0.42 & 202.8 & 70.2 & 222.5 \\
\hline 123 & & & 2.0 & -11.21 & 5.89 & -0.43 & 202.8 & 70.2 & 222.5 \\
\hline 124 & & & 2.1 & -11.21 & 5.89 & -0.45 & 202.8 & 70.2 & 222.5 \\
\hline 125 & & & 2.2 & -11.21 & 5.89 & -0.46 & 202.8 & 70.2 & 222.5 \\
\hline 126 & & 0.20 & 1.8 & -11.21 & 5.89 & -1.61 & 202.8 & 70.2 & 222.5 \\
\hline 127 & & & 1.9 & -11.21 & 5.89 & -1.67 & 202.8 & 70.2 & 222.5 \\
\hline 128 & & & 2.0 & -11.21 & 5.89 & -1.73 & 202.8 & 70.2 & 222.5 \\
\hline 129 & & & 2.1 & -11.21 & 5.89 & -1.79 & 202.8 & 70.2 & 222.5 \\
\hline 130 & & & 2.2 & -11.21 & 5.89 & -1.85 & 202.8 & 70.2 & 222.5 \\
\hline 131 & & 0.40 & 1.8 & -11.21 & 5.89 & -3.21 & 202.8 & 70.2 & 222.5 \\
\hline 132 & & & 1.9 & -11.21 & 5.89 & -3.34 & 202.8 & 70.2 & 222.5 \\
\hline 133 & & & 2.0 & -11.21 & 5.89 & -3.46 & 202.8 & 70.2 & 222.5 \\
\hline 134 & & & 2.1 & -10.28 & 5.10 & -3.02 & 195.0 & 70.2 & 199.1 \\
\hline 135 & & & 2.2 & -10.28 & 5.10 & -3.12 & 195.0 & 70.2 & 199.1 \\
\hline
\end{tabular}

\begin{tabular}{|c|c|c|c|c|c|c|c|c|c|}
\hline \multicolumn{4}{|c|}{ Instance (continued) } & \multicolumn{3}{|c|}{ Variation in $\%$} & \multicolumn{3}{|c|}{ Total number of hours } \\
\hline \# & $\psi$ & $\varphi$ & $\rho$ & Duration & Cost & Quality & Perm. saving & Temporary & Overtime \\
\hline 136 & 0.80 & 0.60 & $\overline{1.8}$ & -9.35 & 4.09 & -3.73 & 210.6 & 70.2 & 189.7 \\
\hline 137 & & & 1.9 & -8.41 & 3.66 & -3.31 & 210.6 & 70.2 & 180.3 \\
\hline 138 & & & 2.0 & -8.41 & 3.66 & -3.44 & 210.6 & 70.2 & 180.3 \\
\hline 139 & & & 2.1 & -8.41 & 3.66 & -3.57 & 210.6 & 70.2 & 180.3 \\
\hline 140 & & & 2.2 & -8.41 & 3.66 & -3.70 & 210.6 & 70.2 & 180.3 \\
\hline 141 & & 0.80 & 1.8 & -7.48 & 2.80 & -3.70 & 187.2 & 46.8 & 165.4 \\
\hline 142 & & & 1.9 & -7.48 & 2.80 & -3.86 & 187.2 & 46.8 & 165.4 \\
\hline 143 & & & 2.0 & -7.48 & 2.80 & -4.02 & 187.2 & 46.8 & 165.4 \\
\hline 144 & & & 2.1 & -5.61 & 2.22 & -3.03 & 124.8 & 46.8 & 103.0 \\
\hline 145 & & & 2.2 & -5.61 & 2.22 & -3.13 & 124.8 & 46.8 & 103.0 \\
\hline 146 & & 1.00 & 1.8 & -5.61 & 2.22 & -3.40 & 124.8 & 46.8 & 103.0 \\
\hline 147 & & & 1.9 & -4.67 & 1.01 & -2.73 & 109.2 & 0.0 & 109.2 \\
\hline 148 & & & 2.0 & -4.67 & 1.01 & -2.87 & 109.2 & 0.0 & 109.2 \\
\hline 149 & & & 2.1 & -2.80 & 0.58 & -1.89 & 62.4 & 0.0 & 62.4 \\
\hline 150 & & & 2.2 & -2.80 & 0.58 & -1.98 & 62.4 & 0.0 & 62.4 \\
\hline 151 & 1.00 & 0.05 & 1.8 & -9.35 & 6.91 & -0.32 & 187.2 & 109.2 & 194.5 \\
\hline 152 & & & 1.9 & -9.35 & 6.91 & -0.33 & 187.2 & 109.2 & 194.5 \\
\hline 153 & & & 2.0 & -9.35 & 6.91 & -0.34 & 187.2 & 109.2 & 194.5 \\
\hline 154 & & & 2.1 & -9.35 & 6.91 & -0.35 & 187.2 & 109.2 & 194.5 \\
\hline 155 & & & 2.2 & -9.35 & 6.91 & -0.36 & 187.2 & 109.2 & 194.5 \\
\hline 156 & & 0.20 & $\overline{1.8}$ & -9.35 & 6.91 & -1.26 & 187.2 & 109.2 & 194.5 \\
\hline 157 & & & 1.9 & -9.35 & 6.91 & -1.31 & 187.2 & 109.2 & 194.5 \\
\hline 158 & & & 2.0 & -9.35 & 6.91 & -1.35 & 187.2 & 109.2 & 194.5 \\
\hline 159 & & & 2.1 & -9.35 & 6.91 & -1.40 & 187.2 & 109.2 & 194.5 \\
\hline 160 & & & 2.2 & -9.35 & 6.91 & -1.44 & 187.2 & 109.2 & 194.5 \\
\hline 161 & & 0.40 & $\overline{1.8}$ & -9.35 & 6.91 & -2.53 & 187.2 & 109.2 & 194.5 \\
\hline 162 & & & 1.9 & -9.35 & 6.91 & -2.62 & 187.2 & 109.2 & 194.5 \\
\hline 163 & & & 2.0 & -9.35 & 6.91 & -2.71 & 187.2 & 109.2 & 194.5 \\
\hline 164 & & & 2.1 & -9.35 & 6.91 & -2.79 & 187.2 & 109.2 & 194.5 \\
\hline 165 & & & 2.2 & -9.35 & 6.91 & -2.88 & 187.2 & 109.2 & 194.5 \\
\hline 166 & & 0.60 & $\overline{1.8}$ & -8.41 & 5.39 & -3.38 & 210.6 & 109.2 & 180.4 \\
\hline 167 & & & 1.9 & -8.41 & 5.39 & -3.51 & 210.6 & 109.2 & 180.4 \\
\hline 168 & & & 2.0 & -8.41 & 5.39 & -3.63 & 210.6 & 109.2 & 180.4 \\
\hline 169 & & & 2.1 & -7.48 & 3.53 & -3.18 & 187.2 & 62.4 & 166.4 \\
\hline 170 & & & 2.2 & -7.48 & 3.53 & -3.30 & 187.2 & 62.4 & 166.4 \\
\hline 171 & & 0.80 & 1.8 & -7.48 & 3.53 & -3.79 & 187.2 & 62.4 & 166.4 \\
\hline 172 & & & 1.9 & -7.48 & 3.53 & -3.94 & 187.2 & 62.4 & 166.4 \\
\hline 173 & & & 2.0 & -7.48 & 3.53 & -4.09 & 187.2 & 62.4 & 166.4 \\
\hline 174 & & & 2.1 & -7.48 & 3.53 & -4.24 & 187.2 & 62.4 & 166.4 \\
\hline 175 & & & 2.2 & -5.61 & 2.96 & -3.23 & 124.8 & 62.4 & 104.0 \\
\hline 176 & & 1.00 & 1.8 & -4.67 & 1.01 & -2.43 & 109.2 & 0.0 & 109.2 \\
\hline 177 & & & 1.9 & -4.67 & 1.01 & -2.57 & 109.2 & 0.0 & 109.2 \\
\hline 178 & & & 2.0 & -4.67 & 1.01 & -2.70 & 109.2 & 0.0 & 109.2 \\
\hline 179 & & & 2.1 & -4.67 & 1.01 & -2.84 & 109.2 & 0.0 & 109.2 \\
\hline 180 & & & 2.2 & -2.80 & 0.58 & -1.85 & 62.4 & 0.0 & 62.4 \\
\hline
\end{tabular}

\section{References}

Afruzi, E. N., Najafi, A. A., Roghanian, E., \& Mazinani, M. (2014). A multi-objective imperialist competitive algorithm for solving discrete time, cost and quality trade-off problems with mode-identity and resource-constrained situations. Computers and Operations Research, 50, 80-96.

Alvanchi, A., Lee, S., \& Abourizk, S. (2012). Dynamics of working hours in construction. Journal of Construction Engineering and Management, 138(1), 66-77.

Babu, A., \& Suresh, N. (1996). Project management with time, cost, and quality considerations. European Journal of Operational Research, 88, 320-327.

Beckers, D. G. J., van der Linden, D., Smulders, P. G. W., Kompier, M. A. J., van Veldhoven, M. J. P., \& van Yperen, N. W. (2004). Working overtime hours: Relations with fatigue, work motivation, and the quality of work. Journal of Occupational and Environmental Medicine, 46, 1282-1289.

Broschak, J. P., \& Davis-Blake, A. (2006). Mixing standard work and nonstandard deals: the consequences of heterogeneity in employment arrangements. Academy of Management Journal, 49(2), 371-393.

Business Roundtable (BRT) (1980). Scheduled overtime effect on construction project, a construction industry cost effectiveness. Procter and Gamble construction projects.

Circadian (2016). Staffing levels: A key to managing risk in 24/7 operations. Circadian Information LP.

Cuyper, N. D., Jong, J. D., Witte, H. D., Isaksson, K., Rigotti, T., \& Schalk, R. (2008) Literature review of theory and research on the psychological impact of temporary employment: towards a conceptional model. International Journal of Management Reviews, 10(1), 25-51.

Fundeling, C. U., \& Trautmann, N. (2010). A priority-rule method for project scheduling with work-content constraints. European Journal of Operational Research, 203 568-574.

Gibson, R. (2015). Practical guide to disruption and productivity loss on construction and engineering projects. Wiley-Blackwell.

Gunduz, M. (2004). A quantitative approach for evaluation of negative impact of overmanning on electrical and mechanical projects. Building and Environment 39(5), 581-587.

Gunduz, M., \& Hanna, A. S. (2005). Benchmarking change order impacts on productivity for electrical and mechanical projects. Building and Environment, 40, 1068-1075.

Hanna, A. S., Chang, C.-K., Lackney, J. A., \& Sullivan, K. T. (2007). Impact of overmanning on mechanical and sheet metal labor productivity. Journal of Construction Engineering and Management, 22-28. 
Hanna, A. S., \& Sullivan, K. T. (2004). Impact of overtime on construction labor productivity. Cost Engineering, 46(4), 20-27.

Hanna, A. S., Taylor, C. S., \& Sullivan, K. T. (2005). Impact of extended overtime on construction labor productivity. Journal of Construction Engineering and Management, 734-739.

Hartmann, S., \& Briskorn, D. (2010). A survey of variants and extensions of the resource-constrained project scheduling problem. European Journal of Operational Research, 207(1), 1-14.

Heimerl, C., \& Kolisch, R. (2010). Scheduling and staffing multiple projects with a multi-skilled workforce. Operations Research, 32, 343-368.

Hitchens, D. M., Wagner, K., \& Birnie, J. E. (1992). Measuring the contribution of product quality to competitiveness: A note on theory and policy. The Economic and Social Review, 23, 455-463.

Kaming, P. F., Holt, G. D., Kometa, S. T., \& Olomolaiye, P. O. (1998). Severity diagnosis of productivity problems: a reliability analysis. International Journal of Project Management, 16, 107-113.

Khang, D. B., \& Myint, Y. M. (1999). Time, cost and quality trade-off in project management: a case study. International Journal of Project Management, 17, 249-256.

Kim, J. Y., Kang, C. W., \& Hwang, I. (2012). A practical approach to project scheduling: considering the potential quality loss cost in the time-cost tradeoff problem. International Journal of Project Management, 30, 264-272.

Kolisch, R., \& Sprecher, A. (1996). Psplib - a project scheduling problem library. European Journal of Operational Research, 96, 205-216.

Li, H., Love, P. E. D., \& Drew, D. S. (2000). Effects of overtime work and additional resources on project cost and quality. Engineering, Construction and Architectural Management, 7(3), 211-220.

Liberatore, M. J., \& Pollack-Johnson, B. (2013). Improving project management decision making by modeling quality, time, and cost continuously. IEEE Transactions on Engineering Management, 30, 518-528.

Mahmud, A. (2015). Effects of worker fatigue on product quality: A case study in apparel industry. International Journal of Scientific and Engineering Research, 6, $1625-1632$.

Mechanical Contractors Association of America (MCAA) (2011). How to estimate the impacts of overtime on labor productivity. MCAA Management Methods Manual, Bulletin No. OT1 Revised, http://tiac.ca/wp-content/uploads/2017/09/IChange-Orders-Productivity-Overtime-A-Primer-for-the-Construction-Industry. pdf.

Mohammadipour, F., \& Sadjadi, S. J. (2016). Project cost-quality-risk tradeoff analysis in a time-constrained problem. Computers and Industrial Engineering, 95, $111-121$.
Mungle, S., Benyoucef, L., Son, Y.-J., \& Tiwari, M. K. (2013). A fuzzy clustering-based genetic algorithm approach for time-cost-quality trade-off problems: A case study of highway construction project. Engineering Applications of Artificial Intelligence, 26, 1953-1966.

Naber, A., \& Kolisch, R. (2014). Mip models for resource-constrained project scheduling with flexible resource profiles. European Journal of Operational Research, 239, 335-348.

National Electrical Contractors Association (NECA) (1989). Overtime and productivity in electrical construction, https://www.smacna.org/resources/resource/2016/ 08/22/neca-productivity-studies.

Orm, M. B., \& Jeunet, J. (2018). Time cost quality trade-off problems: A survey exploring the assessment of quality. Computers and Industrial Engineering, 118, 319-328.

Pencavel, J. (2014). The productivity of working hours. The Economic Journal, 125, 2052-2076.

Rojas, E. M. (2008). Construction productivity: A practical guide for building and electrical contractors. J. Ross Publishing.

Russell, A., \& Taghipour, S. (2019). Multi-objective optimization of complex scheduling problems in low-volume low-variety production systems. International Journal of Production Economics, 208, 1-16.

Stadler, W. (1988). Multicriteria optimization in engineering and in the sciences. Springer Science+Business Media, LLC.

Tareghian, H. R., \& Taheri, S. H. (2006). On the discrete time, cost and quality trade-off problem. Applied Mathematics and Computation, 181, 1305-1312.

Thomas, H. R., \& Raynar, K. A. (1997). Scheduled overtime and labor productivity: quantitive analysis. Journal of Construction Engineering and Management, 181-188.

U.S. Army Corps of Engineers (1979). Modification Impact Evaluation Guide EP 415-1-3. Department of the Army.

Weglarz, J., Jozefowska, J., Mika, M., \& Waligora, G. (2011). Project scheduling with finite or infinite number of activity processing modes: A survey. European Journal of Operational Research, 208, 177-205.

Yang, B., Guenes, J., \& Brien, W. J. O. (2004). A heuristic approach for minimizing weighted tardiness and overtime costs in single resource scheduling. Computers and Operations Research, 31, 1273-1301.

Zhang, L., Du, J., \& Zhang, S. (2014). Solution to the time-cost-quality trade-off problem in construction projects based on immune genetic particle swarm optimization. Journal of Management in Engineering, 30, 163-172. 